\title{
Thermal Behaviour and Refrigeration of High-Temperature Superconducting Fault Current Limiters and Microlimiters
}

\author{
J.A. Veira, M.R. Osorio* and F. Vidal \\ LBTS, Departamento de Física da Materia Condensada, \\ Universidade de Santiago de Compostela, Santiago de Compostela
}

Spain

\section{Introduction}

Superconducting fault current limiters (SFCL) based on high temperature superconductors (HTSC) of different types, inductive, resistive or hybrid, have being extendedly studied (Noe \& Steurer, 2007; Sokolovsky et al., 2004; Paul et al., 2001) and tested in power plants (Paul et al., 1997; Leung et al., 1997; Zueger et al., 1998). Inductive SFCL, intended to operate at very high powers, are in general based on bulk HTSC whereas resistive or hybrid types are based on both bulk (mainly in the shape of bars) and thin films grown on different types of substrates and mainly patterned in meandered paths. HTSC based limiters represent one of the more promising applications of such materials but both bulk and thin film HTSC present different drawbacks which make difficult the practical implantation of these devices. Important problems concern, in particular, to the thermal behaviour of samples under current faults. In bulk samples, which are quite inhomogeneous, specially the huge elements required for limiters operating at high powers, overcurrents under fault generate hot spots in weak zones which endanger their thermal stability and lead to an irreversible damage or to an unsatisfactory performance of the device (low impedance, long recovery times once the fault is cleared, etc.).

In terms of thermal behaviour, thin films could show a better performance than bulk samples. Their critical current density and electric field above their normal-superconducting transition are around two orders of magnitude higher than those for bulk samples. Accordingly, the heat developed per unit volume under a current fault is also orders of magnitude higher. This dissipation, together with the small thickness of films, should provoke a homogeneous and full transition of the overall sample showing, therefore, a very fast and abrupt increase of the resistance (Antognazza et al., 2002). In addition, the large surface-to-volume ratio of thin film samples, the low boundary thermal impedance film-substrate and the great thermal conductivity of substrates determine a very good heat evacuation once the current fault is cleared. Hence the recovery times observed in limiters based on thin films are much shorter than for limiters based on bulk samples (Osorio et al., 2006). In what concerns the transition to the normal state it may occur, however, that indeed only some parts of the film meander

*Present address: Laboratorio de Bajas Temperaturas, Departamento de Física de la Materia Condensada, Universidad Autónoma de Madrid, Spain. 
are triggered to the normal state by the current fault (Paul et al., 2001). In that case dissipation can be so concentrated in some of these parts that films could also be irreversibly damaged. To prevent that some weak parts of the long meander thin film paths be burning out, it has been proposed to fabricate these elements by patterning a set of constrictions alternated with wider portions. With this design, the normal transition would formerly occur in the narrower segments and the heat arising from this overheated sections will rapidly propagate along the whole meander path (i.e. including also the wider parts), hence leading to a complete and more homogeneous transition (Decroux et al., 2003; Antognazza et al., 2005; Duron et al., 2005; 2007; Antognazza et al., 2007; Duron et al., 2007).

For both bulk and thin film based SFCL the thermal behaviour and refrigeration of the superconducting elements determine, therefore, the overall performance of these devices. In this work we will summarize some of our results on this issue including the thermal recovery of inductive SFCL operating with bulk cylinders of different sizes (Osorio et al., 2006) or with artificial weak zones (Osorio et al., 2005; 2008), the improvement obtained by using stacks of bulk rings or thin film washers as secondary (Osorio et al., 2004; 2006), the use of thermoacoustic refrigerators (Osorio et al., 2008) and the possibility of using thin film microbridges as very efficient microlimiters in very low power applications (Lorenzo et al., 2009).

\section{Bulk cylinders as secondary of inductive SFCL}

Despite the advances in the fabrication of HTSC materials the presence in the samples of inhomogeneities which act as weak zones still represents an important drawback for their use in SFCL. The presence of weak zones provokes an earlier transition to a dissipative state of only a part of the samples that, as indicated above, can cause a great degradation of the performance of the overall SFCL device. In the first part of this section we will summarize our results on the non-homogeneous quench of bulk Bi-2223 cylinders acting as secondaries of inductive SFCL, in particular, on the current limitation capacity under fault of the limiters and their thermal recovery once the current fault is cleared. In the second part we will outline our numerical approaches to account for the effects of the bi-phase character of Bi-2223 samples on the quench under a current fault.

\subsection{Non-homogeneous quench of the superconducting secondary of inductive SFCL}

\subsubsection{Samples' characteristics}

In our studies of inductive SFCL based on bulk HTSC we have used samples of nominal composition $\mathrm{Bi}_{1.8} \mathrm{~Pb}_{0.26} \mathrm{Sr}_{2} \mathrm{Ca}_{2} \mathrm{Cu}_{3} \mathrm{O}_{10+x}$. This samples were obtained from cylinders of 120 $\mathrm{mm}$ in height, $21 \mathrm{~mm}$ in internal diameter and $2.4 \mathrm{~mm}$ in wall thickness supplied by Can Superconductors (Czech Republic). Commercial samples were cut in shorted cylinders and rings, around $40 \mathrm{~mm}$ and $5 \mathrm{~mm}$ in height, respectively, to carry out our measurements. Also, other small pieces were properly cut for material characterization: electrical resistivity, characteristic voltage-current curves (CVC), magnetic susceptibility and x-ray diffraction. The results of our characterization tests show a very good reproducibility for different pieces of the same commercial cylinders and also for pieces obtained from different cylinders.

Typical examples of electrical resistivity versus temperature curves, $\rho(T)$, CVC and magnetic susceptibility $\chi(T)$ are displayed in figures 1 to 3 . In figure 1 the dotted line represents the linear fir to the normal state resistivity data. In the inset it is shown the width of the 


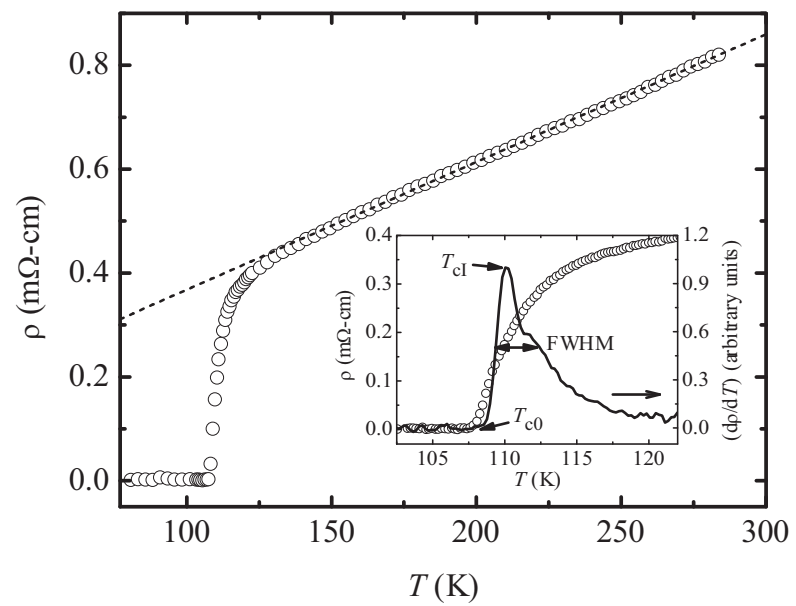

Fig. 1. Resistivity as a function of temperature for a piece of the material employed as secondary of the SFCL. Inset: detailed view of the superconducting transition. The solid line represents the temperature derivative (on arbitrary units). The critical temperatures $T_{\mathrm{CI}}$ and $T_{\mathrm{c} 0}$ are defined as the ones corresponding to the maximum of the derivative and the minimum value for which $\rho \neq 0$, respectively. The full width half maximum (FWHM) is the width of the derivative at half height. Figure from Ref. (Osorio et al., 2004).

transition, defined as the width of the derivative $d \rho / d T$ (solid line) at medium height. $T_{c I}$ the temperature at which $d \rho / d T$ presents its maximum, and $T_{c 0}$, the minimum temperature for which $\rho \neq 0$ are indicated. In the CVC shown in figure 2, symbols represent the experimental data measured at the indicated temperatures and the solid lines the fits to the usual power law function (Prester, 1998; Osorio et al., 2004). In figure 3 circles correspond to the field cooling (FC) of the sample and squares to its zero field cooling (ZFC).

X-ray diffraction analyses (Osorio et al., 2004) show the presence in our samples of two phases: around $15 \%$ of Bi2212 and $85 \%$ of Bi2223. In $\rho(T)$ or CVC it cannot be distinguished any effect provokated by this inhomogeneity, as may be expected because of the percolative nature of electrical transport properties. It is, however, well visible in the $\chi(T)$ curves. As can be seen in the FC and ZFC measurements of figure 3, the normal-superconducting transition occurs in two steps: one around $108 \mathrm{~K}$ (corresponding to Bi2223 phase) and the the other around $98 \mathrm{~K}$ (corresponding to Bi2212 phase). We will se below that to numerically account for the electrical resistance and temperature of our samples operating as secondaries in our inductive SFCL under current fault, the bi-phase nature of the material plays a central role.

\subsubsection{Samples' behaviour under a current fault}

The electrical resistance and temperature evolution under a current fault of cylinders or rings when acting as secondaries in an inductive limiter was measured by attaching to the samples some voltage contacts and thermocouples along their perimeter as indicated in figure 4 . Details on the limiter configuration and global experimental set up can be seen in reference (Osorio et al., 2004). In figures 5 and 6 we show typical results for this type of test. In this example data were measured by using a $5 \mathrm{~mm}$ in height ring with five couples of voltage 


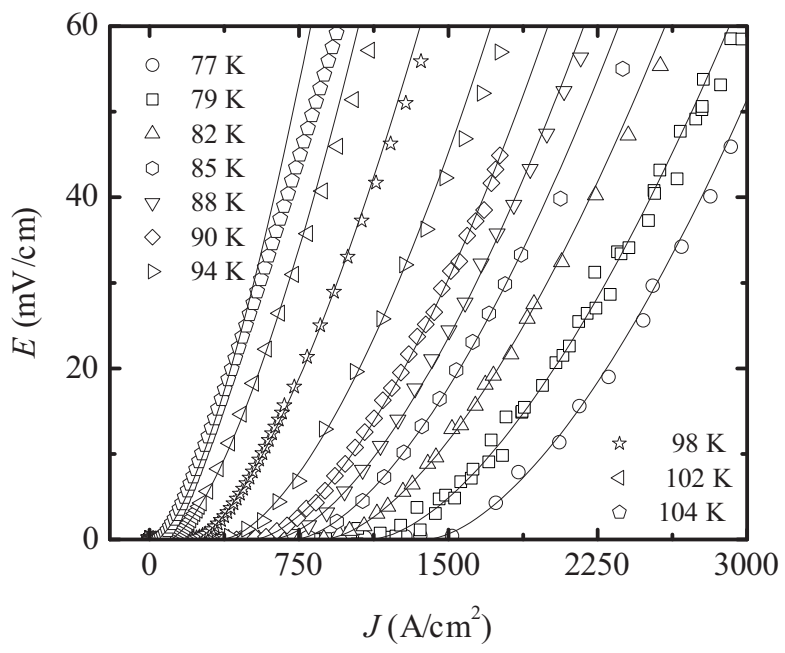

Fig. 2. $E-J$ curves measured in a piece of the superconducting elements employed in the limiter. The solid lines represent the fittings to the usual power law function (Prester, 1998). Figure from Ref. (Osorio et al., 2004).

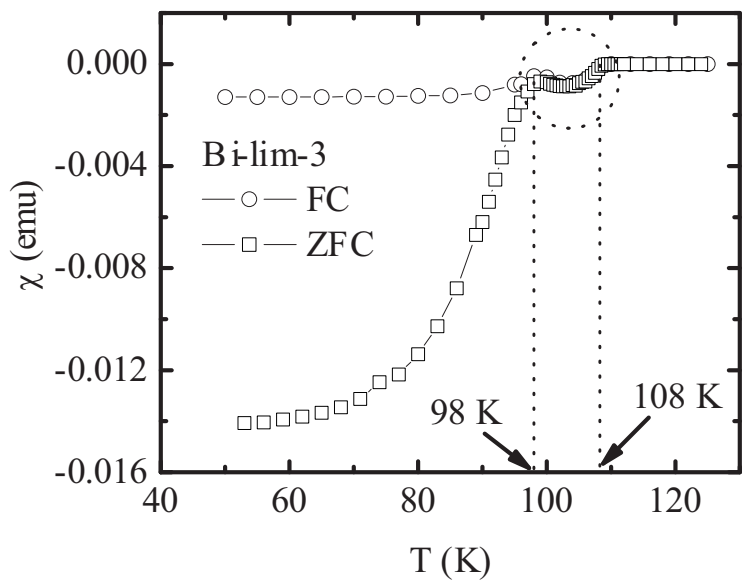

Fig. 3. Variation with temperature of the magnetic susceptibility during field cooling and zero field cooling for a piece of the cylinder used as secondary of the limiter. Notice the presence of two superconducting phases, both identified by their own critical temperatures. Figure from Ref. (Osorio et al., 2004). 


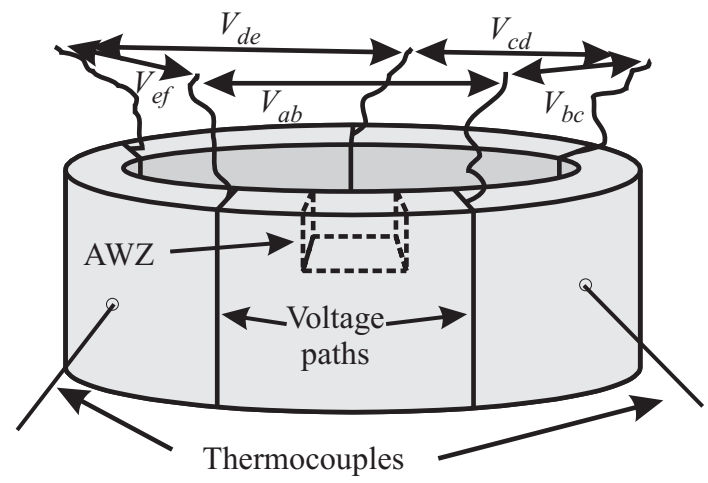

Fig. 4. Scheme of division into regions (five in this example) of a ring with voltage paths and thermocouples attached on each region. The dashed lines indicate the artificial weak zone made by reducing the cross section of the ring in a small portion of its perimeter. Figure from Ref. (Osorio et al., 2004).

contacts and five thermocouples. Figure 5 shows the measured wave-forms. For clarity we use lines instead of symbols and represent only three of the five signals.

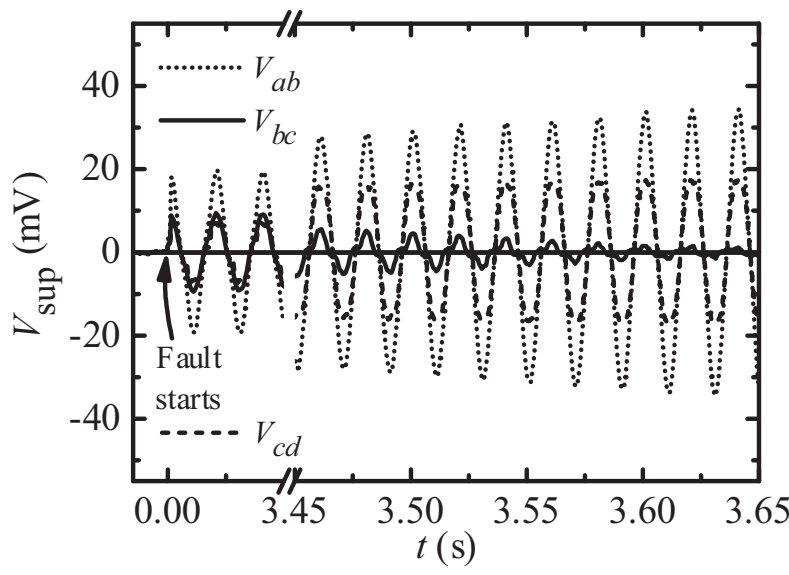

Fig. 5. Voltage measured during a fault with the arrangement depicted in figure 4 . The different regions do not behave in the same way, as one of them cools down while others enter in the ohmic state. Figure from Ref. (Osorio et al., 2004).

In figure 6(a) it is shown the rms values of the five voltage signals and in figure $6(\mathrm{~b})$ the corresponding temperature readings. We can observe that the voltage signals of some zones grow continuously during the fault but others experience the inverse tendency, so the voltage is progressively reduced up to typical values of the flux-flow regime and even up to zero indicating that these zones return to the superconducting state. The corresponding temperatures, displayed in figure 6(b), also indicate this tendency: the parts at high voltage heat up to temperatures well above $T_{C I}$ whereas the parts at low voltage scarcely heat up remaining at temperatures below $T_{c 0}$. In this sample, for the region denoted $c d$, the temperature reading is lower that the one that could be expected by inspecting the rms 


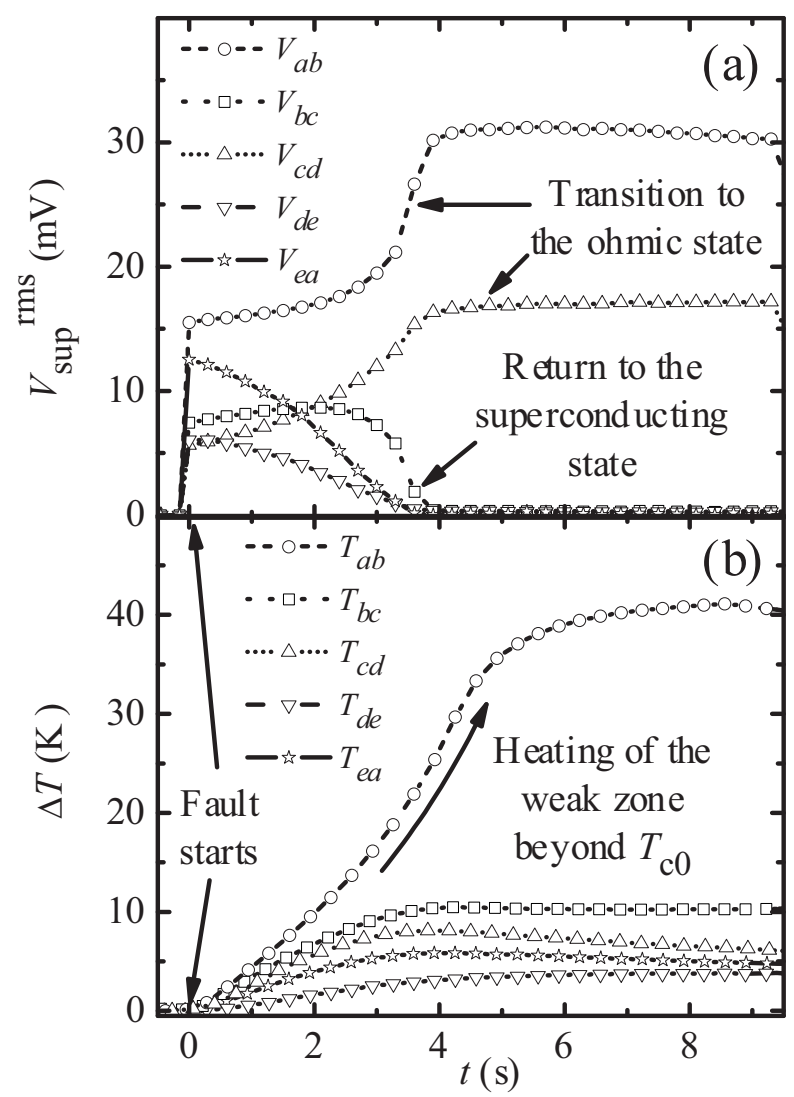

Fig. 6. (a) Rms voltages for all the considered regions of the ring of figure 4 . The major part of the sample recovers the superconducting state as the current is limited by the dissipative zones. (b) Temperature excursions obtained for an identical fault. As it can be seen, it exists a remarkable coherence between these results and the voltage measurements. See text for details. Figure from Ref. (Osorio et al., 2004).

voltage. This disagreement could be due to the presence of a relatively small thermal inhomogeneity in this zone, or to an inhomogeneity located in depth, relatively far away from the point at which the thermocouple was attached. In all the studied rings it was found a voltage signal behaviour like the one followed by the voltages denoted $a b$, clearly due to the thermal evolution during the fault of the more dissipative zone in each sample.

The results obtained in this type of test are very similar to those of figure 7 measured by using a ring in which a weak zone was created by reducing its height somewhere (as indicated by dotted lines in figure 4). The symbols represent the temperatures measured with thermocouples attached at the weak zone and at points at $\pm 1 \mathrm{~cm}$ and $\pm 2 \mathrm{~cm}$ far away along the ring perimeter. When the fault is provoked, so the induced current overtakes the critical current, $I_{\mathrm{c}}$, the whole superconductor enters in the dissipative state, but the temperature 


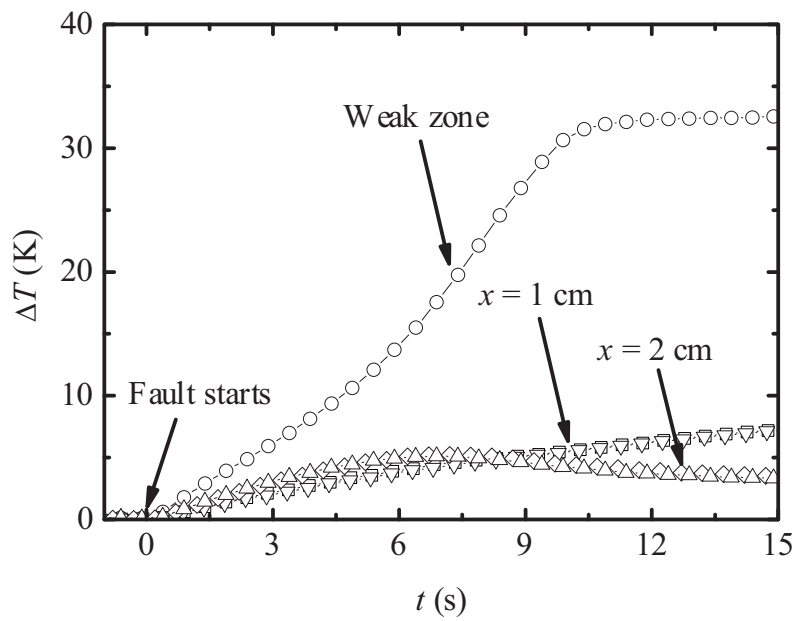

Fig. 7. Temperature excursions when provoking the transition (by exceeding the critical current) of the ring of figure 4 (once the AWZ has been made). The readings of the thermocouples located $1 \mathrm{~cm}$ away from the hot spot are denoted by down-triangles and squares, and the ones for $2 \mathrm{~cm}$ away by up-triangles and diamonds. Notice that, while the majority of the ring heats up scarcely, and it remains very far from the ohmic state, the dissipative zone (circles) warms up till around the critical temperature. Then it is reached a stable plateau till the end of the fault at $t=30 \mathrm{~s}$. The fact that, during the first seconds, the temperature increment is slightly greater at $x=2 \mathrm{~cm}$ than at $x=1 \mathrm{~cm}$ can be due to the local inhomogeneities. Figure from Ref. (Osorio et al., 2004).

increment in the majority of the ring is quite low, as the thermocouples indicate, and only in the weak zone it is reached the critical value, $T_{c}$. In fact, the temperature increment far away from the weak zone should be due to heat coming from the dissipative zone, instead of being generated in the corresponding parts of the sample. This fact would explain why at $1 \mathrm{~cm}$ away from the centre of the weak zone the temperature increases slowly when it has been already reached a stable plateau in the hottest region. Notice that the showed behaviour, with the decreasing temperature in the further parts of the ring, means that the warm zone does not tend to expand itself, but remains essentially still till the end of the fault (at about $t=30 \mathrm{~s}$ ). This fact is in part a consequence of the reduction of the induced current, due to the gain in impedance originated by the very resistive zone. As the current that circulates in the ring diminishes, the dissipation becomes lower, and so the thermal excursion in the majority of the sample. This fact provokes that the resistance of the superconductor, and so the total impedance of the limiter, increase quite smooth.

\subsection{The effects of the bi-phase character of samples on the quench}

The behaviour of inductive SFCL is mainly determined by the overheating of the weak zone in the superconducting cylinder. In turn, the behavior of this zone seems to be mainly dominated by the minority phase Bi-2212, that has the lower critical temperature $T_{c 0} \approx 98 \mathrm{~K}$, as indicates the change of slope observed in the voltage curves of figure $6(\mathrm{a})$, which corresponds to $\Delta T \approx$ $20 \mathrm{~K}$, i.e. at $T \approx 98 \mathrm{~K}$, in the temperature versus time curves plotted in figure $6(\mathrm{~b})$. 
The role of the minority phase in the thermal evolution of the superconducting element during a fault can be illustrated by using a very simple approach for the temperature distribution in the superconductor. The calculations are done by using an iterative numerical routine in Matlab (The MathWorks, Inc, USA). The magnetic circuit is represented through field variable inductance coefficients (Osorio et al., 2004; MITStaff, 1961; Paul et al., 1995) and the superconducting element is modeled by taking into account the experimental characterization data, as it will be described later. The input variable is the applied voltage, kept as constant, and the fault is simulated by reducing the impedance of the circuit. During the iterative process, the circulating currents $\left(I_{\mathrm{p}}\right.$ and $\left.I_{\mathrm{sup}}\right)$ are first calculated at a time $t$, and then the resistivity and the temperature increment of the superconducting element. These steps are repeated till it is reached a set of self-consistent values, so the routine advances to time $t+\delta t$ and so on.

We suppose that the cylinder is divided into two temperature regions, as indicated in figure 8. The region around the weak zone is considered to be at a higher temperature (hot domain) than the remainder part of the cylinder (cold domain). The length, temperature and resistance of each domain are denoted, respectively, by $\ell^{h}, T^{h}, R^{h} \equiv\left(\rho^{h}\left(I_{\text {sup }}, T^{h}\right) \ell^{h}\right)\left(A_{\perp}\right)^{-1}$ and $\ell^{c}$ $\left(=2 \pi r-\ell^{h}\right.$, being $r$ the radius of the cylinder $), T^{c}, R^{c} \equiv\left(\rho^{c}\left(I_{\text {sup }}, T^{c}\right) \ell^{c}\right)\left(A_{\perp}\right)^{-1}$, where $\rho^{(c, h)}$ represents the resistivity of each domain and $A_{\perp}$ the cylinder cross section.

The temperature evolution was calculated by applying to both regions the heat balance

$$
\varrho A_{\perp} \ell^{(c, h)} C_{\mathrm{p}}\left(T^{(c, h)}\right) \frac{\partial T^{(c, h)}}{\partial t}=\dot{\mathcal{W}}-\dot{\mathcal{Q}}
$$

$\dot{\mathcal{W}}$ and $\dot{\mathcal{Q}}$ being the power dissipated inside each superconducting region and the convective flux through their surfaces, respectively, which can be expressed as

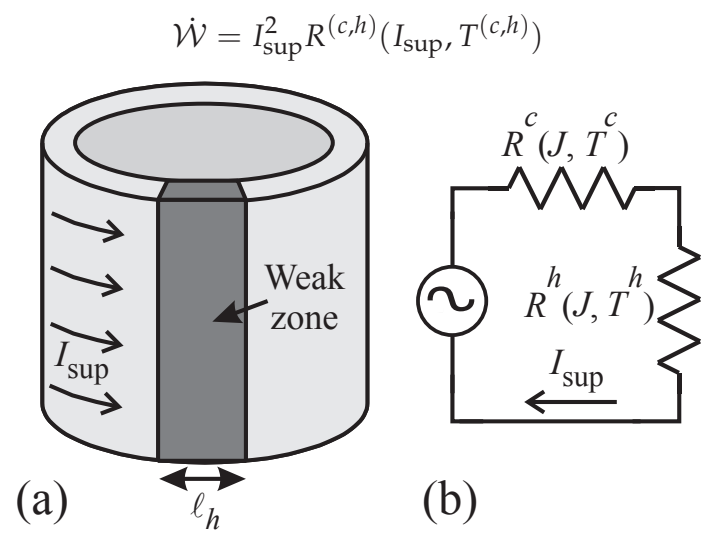

Fig. 8. (a) Scheme of the model supposed for an inhomogeneous superconducting cylinder, which is divided into two regions. The dark one is the hot domain, denoted by the index $h$ (for the cold one stands $c$ ), which it is supposed to have a defect that generates a more dissipative zone. Notice that this region occupies only a small portion of the cylinder perimeter, but its whole height, so the current that goes through both parts is the same. (b) Equivalent circuit of the inhomogeneous superconductor. The resistances of both parts are joint in series. Figure from Ref. (Osorio et al., 2004). 
and

$$
\dot{\mathcal{Q}}=h P \ell^{(c, h)}\left(T^{(c, h)}-T_{\mathrm{b}}\right),
$$

where $\varrho$ is the density of the material, $C_{p}(T)$ its heat capacity (Xiao et al., 1999), $h$ the convective factor, which can be taken as temperature independent with a small error, $T_{b}$ is the environmental temperature and $P$ is the cross section perimeter of the cylinder. The circulating current, $I_{\text {sup }}$, depends on the resistances, $R^{(c, h)}$, and so on the length of the hot domain, $\ell^{h}$, which is set as a free parameter in our calculations. It has been also supposed, for the sake of simplicity, that during the fault the heat flow along the cylinder is negligible, in agreement with the low thermal conductivity of this material and with our experimental results.

The lines plotted in figure 9 represent the temperatures determined in this scenario. The experimental data are those corresponding to the $a \mathrm{~b}$ region in figure 6 . The dashed and short-dashed lines were obtained in the framework of the effective-medium-theory (Osorio et al., 2004; Davidson \& Tinkhan, 1976), considering that the effective electrical resistivity, $\rho_{\mathrm{e}}$, of each domain can be approached according to the concentrations of the majority and the minority phases $\left(C_{1}\right.$ and $C_{2}$, respectively), the depolarization factor for the minority phase inclusions $(X)$ and the corresponding resistivities, for which it is used the relations

$$
\begin{array}{r}
\rho(J, T)=\frac{E(J)}{J} \text { for } T<T_{\mathrm{c} 0} \\
\rho_{\text {normal }}(T) \text { for } T \geq T_{\mathrm{c} 0},
\end{array}
$$

and with $\rho_{\text {normal }}(T)=103+2.3 T \mu \Omega-c m$ (i.e., the same normal resistivity displayed in figure 1). The value of the critical temperature, $T_{\mathrm{c} 0}$, must be chosen depending on the corresponding phase.

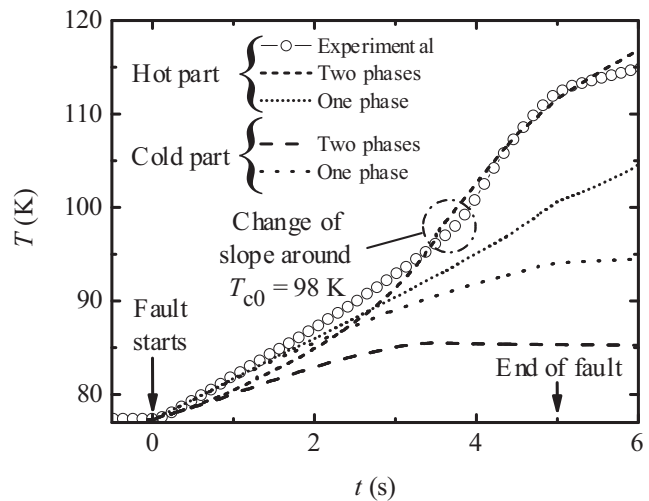

Fig. 9. Temperature excursions simulated when it is supposed that it exists a hot spot created by a slightly different resistivity in a $10 \%$ of the perimeter of the cylinder. It is considered two cases: in the first one the superconductor is a pure Bi-2223 phase, while in the second one it is also considered as a Bi-2223 matrix with Bi-2212 inclusions of spherical shape homogeneously distributed in the sample. It is also included the experimental data for the temperature excursion of the $a \mathrm{~b}$ region of figure 6 , which allows fixing the free parameters of the model (i.e. the resistivity and the length of the weak zone). Figure from Ref.

(Osorio et al., 2004). 
In our calculations (Osorio et al., 2004) we have used the values $X=\frac{1}{3}$ (i.e., we assume that the inclusions are spherical), $C_{1}=0.85$ and $C_{2}=0.15$, in accordance with the x-ray analysis already presented. We have also left the resistivity of the hot domain to be slightly higher than the one of the cold part at each temperature. For that, for $\rho_{\mathrm{e}}^{h}$, we have used $\rho_{\mathrm{e}}^{h}\left(T^{h}\right)=$ $\alpha \rho_{\mathrm{e}}^{c}\left(T^{h}\right)(\alpha \geq 1)$ and carried out the calculations with $\alpha$, altogether with the length $\ell^{h}$, as free parameters. The dashed and short-dashed lines of figure 9 correspond to the best fit with $\alpha=1.01$ and $\ell^{h}=0.1 \times 2 \pi r$. We must note that the precise values of $C_{1}, C_{2}$ and $X$ do not change the main results from our approach. Small variations of these parameters should be balanced by the free ones used in our calculations.

As it can be seen, the general behaviour of the thermal evolution of the hot part is roughly accounted for, in particular the slope changes of the $T(t)$ curve during the fault. The thermal excursion calculated for the cold part of the cylinder is also in good agreement with the data displayed in figures 6 and 7 measured in the parts far away from the weak zones. The dotted lines in figure 9 represent the thermal evolution of the hot (short-dotted) and cold (dotted) parts, calculated by assuming a cylinder composed of only Bi-2223 phase. Clearly, in this scenario the experimental data are not properly accounted for.

Despite the crudeness of our approach, our results indicate that the thermal evolution of the superconducting cylinder during a fault is mainly determined by the minority phase. This behaviour represents one of the most important drawbacks of the inhomogeneous materials to be used for fault current limiter devices, as the limitation can be carried out by only a small part of the superconducting element. This would imply a useless waste of material and, for high values of the critical current density, a huge dissipation in the surroundings of the weakest parts which could lead to a further degradation or even to a local melting. Therefore, work should be done in order to improve the homogeneity of the superconducting samples employed in SFCLs or by designing special configurations which allow to reduce the importance of this problem.

We must finally note that the global resistance of the cylinder, obtained according to the scheme of figure $8(\mathrm{~b})$ by using the expression

$$
R\left(J, T^{c}, T^{h}\right)=\rho_{\mathrm{e}}^{c}\left(J, T^{c}\right)\left(\frac{\ell^{c}}{A_{\perp}}\right)+\rho_{\mathrm{e}}^{h}\left(J, T^{h}\right)\left(\frac{\ell^{h}}{A_{\perp}}\right),
$$

roughly reproduces the experimental data (Osorio et al., 2004).

In figure 10, symbols represent the temperature measured in the weak zone during the thermal recovery. The lines represent the temperature determined with our numerical routine, which in this case is, in principle, very simple. The current is set to zero and the temperature is let to vary according to the convective exchange of heat, dashed line, or to a convective plus conductive model (to the neighbouring cold parts), solid line. It is well noticeable that a conductive exchange term between the weak zone and the rest of the cylinder must be included to account for the temperature diminution.

\section{Thermal behavior of an inductive SFCL whose secondary has artificial weak zones}

The results shown in figure 10 indicate that the convection mechanism is not enough to explain the cooling down of the weak zone once the fault is cleared, but conduction to the cold parts 


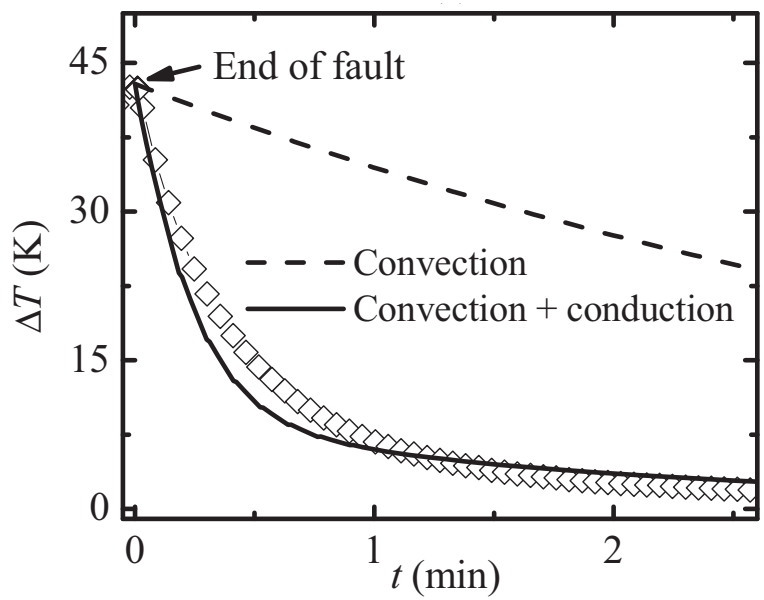

Fig. 10. Temperature measured in the weak zone during the thermal recovery of the bulk cylinder under study. The dashed line shows the calculation for the pure convection model (exponential decrease), while the solid line takes into account not only the convection term, but also a conductive exchange between the weak zone and the rest of the cylinder. Figure from Ref. (Osorio et al., 2005).

of the cylinder plays a major role. Notice that this would entail that this weak zone would refrigerate faster than a homogeneous cylinder, provided that the same final temperature is attained in both cases. This suggests that if the cylinder was made up from weak zones separated by cold segments, after the fault clearance the excess heat could be removed from the hot parts to the colder ones, till the cylinder was thermalized at a low temperature, maybe higher than that of the coolant, but much lower than the maximum value obtained during the fault. It can be argued that, as only the weak parts would enter into the normal state, the impedance of the limiter should be much lower. Nevertheless, and despite this drawback, it could be found a configuration of made-to-measure weak zones, properly spaced, which allow obtaining an acceptable impedance but, in turn, would reduce the recovery time to a competitive value, even in non-liquid environments.

The above ideas were proposed in previous works (Osorio et al., 2005; 2008; 2006), where two possible procedures where stated; in the first one (Osorio et al., 2005), the set of artificial weak zones (AWZ) could be made from a different material, with a lower $J_{c}$ and a higher resistivity (so somewhat balancing the loss of impedance of the limiter), as depicted in figure 11(a), for a set of $n$ AWZ of length $\ell_{n}$, $\ell^{h}$ being the total weak length. Figure 11(b) shows the equivalent electrical circuit for the cylinder, with resistances $R^{c}$ and $R^{h}$ for the cold and hot parts (i.e. the AWZ), respectively.

In the second procedure (Osorio et al., 2008; 2006), each AWZ would be made as a constriction of the cross-section of the cylinder as it is shown in figure 14(a). This configuration allows using the same material for the whole sample, and the reduction in the critical current and the gain in the total impedance is easily yielded by the diminution of the cross-section. For convenience, this idea was initially tested by means of a 1-D numerical simulation routine. This implies assuming that the vertical axis of the cylinder is long enough to neglect 

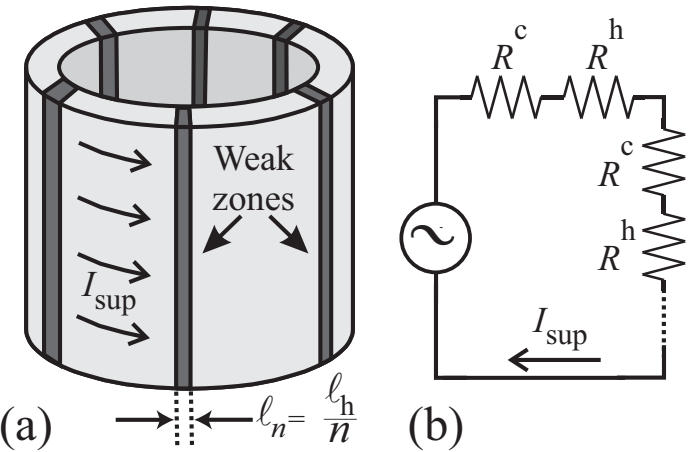

Fig. 11. (a) Scheme of the superconducting cylinder when several thin weak zones are considered instead of a greater size one. The length of each weak zone, $\ell_{n}$, is the total one, $\ell^{\mathrm{h}}$, divided by the number of slices, $n$. (b) Equivalent electrical circuit of the inhomogeneous superconducting cylinder. The resistances are both dependent on the current density and the temperature of the corresponding zone: $R^{(\mathrm{c}, \mathrm{h})}\left(J, T^{(\mathrm{c}, \mathrm{h})}\right)$. Figure from Ref. (Osorio et al., 2005).

any possible temperature gradient along this direction. In addition, it is assumed that the temperature across the wall thickness is approximately the same no matter the point under consideration, i.e., the surface of the cylinder is not at a lower temperature than the inner parts. This is the rougher approximation of the model, but for cylinders of wall thickness of the order of $2-3 \mathrm{~mm}$ it can be quite acceptable. The AWZ are parametrized by the relative length $\lambda$, which is the ratio of the length of the weak zone to the whole perimeter of the cylinder.

Figure 12(a) shows the recovery time calculated for different values of $\lambda$ normalized to that obtained for a homogeneous cylinder (left axis) as well as the normalized impedance (right axis). Notice two important facts; first of all, the recovery time is not necessarily shorter for a cylinder with a weak zone, unless the AWZ is short enough to warranty a fast evacuation of the heat by conduction. Secondly, the impedance yielded by the AWZ can be lower than that offered by the homogeneous cylinder. This second drawback can be removed, in principle, by using a more resistive material or by making AWZ in the shape of grooves (the lower the cross-section the higher the impedance). Even if the resulting impedance is not as high as that offered by the homogeneous cylinder, this inconvenient could be balanced by a much faster thermal recovery. The solution to the first drawback appears to be splitting the AWZ in short slices, so that the heat can be easily removed by conduction from the cold parts to the colder segments which act as heat reservoirs. This effect is shown in 12(b), where an AWZ of length $\lambda=2$ (corresponding to the working point chosen in (a), for which the weak zone represents $20 \%$ of the perimeter of the cylinder) is split into $n$ slices. Notice that, although the weak zones are individually shorter, the total "weak length" is the same, so the impedance can be considered as invariable with respect to the case of $n=1$ (i.e. a non-split weak zone). Maybe a better refrigeration of these slices could lead to a slightly lower impedance, but this effect is neglected here.

Figure 13 shows the numerical calculations for the performances of different cylinders acting as secondary of a SFCL. The first one is a homogeneous element, the second a cylinder with a connected weak zone of relative length $\lambda=2$, and the last one is a cylinder with a split 

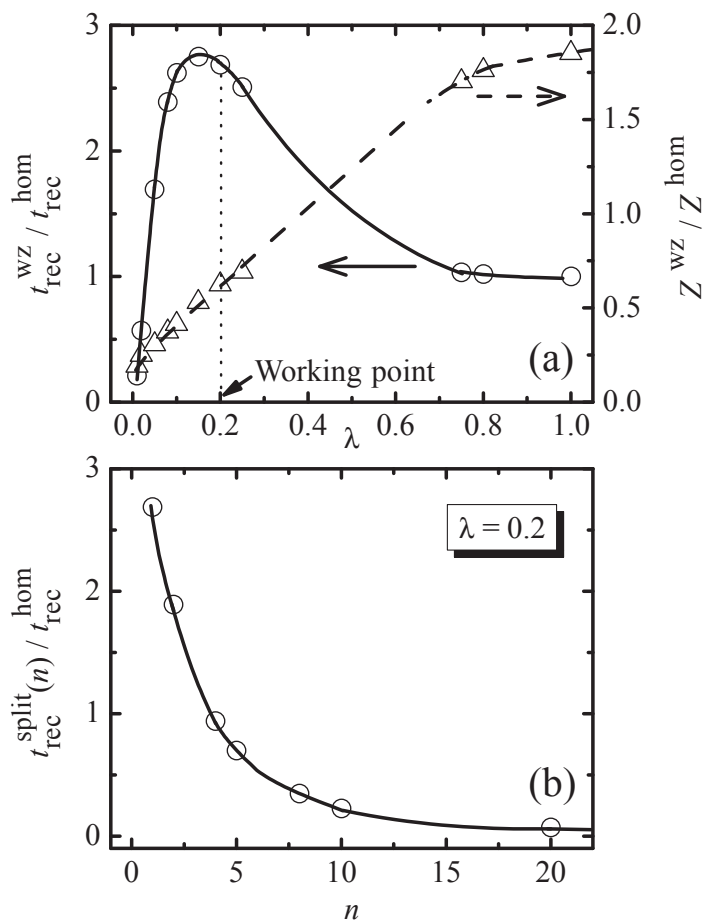

Fig. 12. (a) Recovery time calculated for a set of values of $\lambda$ normalized to the value obtained for a homogeneous cylinder (circles). The right axis shows the normalized impedance (triangles). Both solid and dashed lines are given as guides for the eyes. (b) Recovery time as a function of the number of slices in which it is divided a weak zone with $\lambda=0.2$, normalized to the value when the cylinder is fully homogeneous. The solid line is again a guide for the eyes. Figure from Ref. (Osorio et al., 2005).

weak zone characterized by the same relative length and $n=10$. In figure 13(a) it can be seen the heating and thermal recovery for a fault of about $0.2 \mathrm{~s}$ in duration. Notice that the final temperature difference is about $30 \mathrm{~K}$ in the case of the homogeneous cylinder, while the AWZ of the second and third cylinders reach the same maximum value, about two times higher. Due to this, the recovery of the connected weak zone is even slower than that of the homogeneous cylinder. However, when the AWZ is split into 10 parts the thermal recovery is noticeably enhanced. Figure 13(b) shows the impedance obtained with these cylinders. The homogeneous cylinder yields a much higher impedance, as the transited length is $80 \%$ higher. This drawback can be somewhat balanced by using AWZ made form a material with a higher resistivity (see dashed line, for a material with a normal resistivity two times higher than that of the rest of the superconducting element).

Further tests were realized by means of a finite element routine which allows exploring the effects associated with thick walls. Figure 14 (a) and (b) shows an scheme of the top and the lateral view, respectively, of the cylinder with constrictions equally spaced used in the 

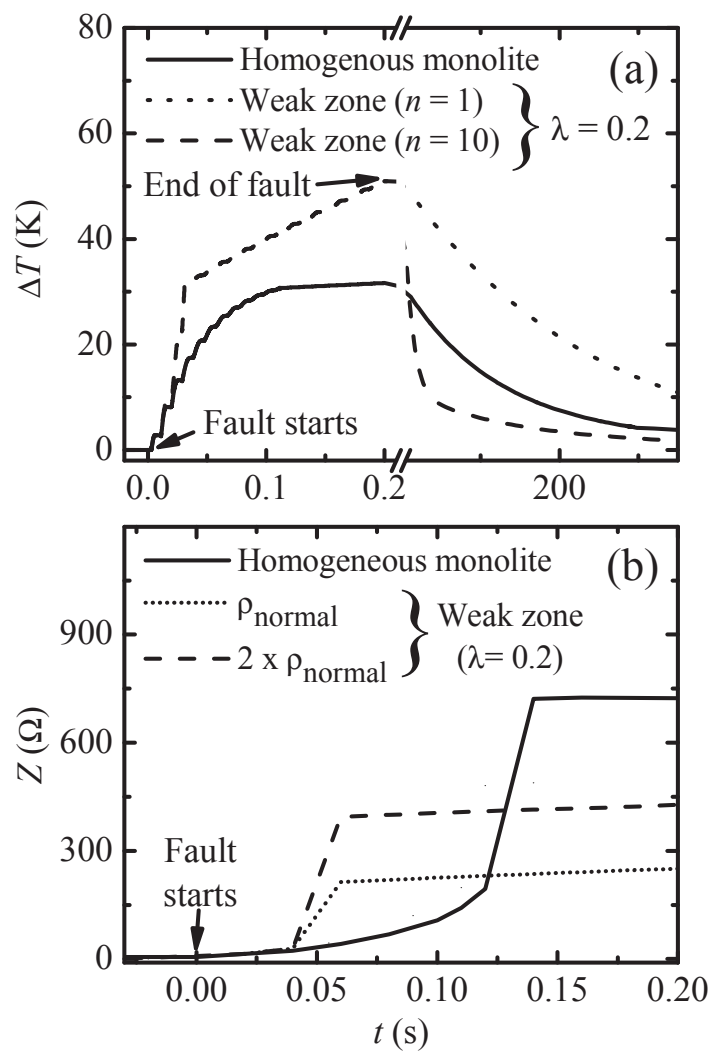

Fig. 13. (a) Temperature excursions calculated for a homogeneous monolith-type cylinder (solid line), a cylinder with a connected weak zone (dotted line) and a cylinder with a weak zone split into 10 small parts (dashed line). The recovery time of the sample with the split weak zone is noticeable shorter than that for the homogeneous sample. In this example, the resistivity of the weak zone is assumed to be 2 times the resistivity of the reminder part. (b) Impedance under a current fault calculated for the device operating with each one of the samples considered in (a). The impedance of the device with the inhomogeneous sample (the same for the connected and the split weak zone) is around a $40 \%$ lower than with the homogenous one, except for the first cycles. The dotted line should be the impedance if the resistivity is the same for both the weak zone and the other part of the cylinder. Figure from Ref. (Osorio et al., 2005).

simulation. It is assumed that a constant current circulates along the circumferential perimeter of a cylinder. For the sake of simplicity, the cylinder is considered to be infinite along its axis, hence the problem is 2-D. Due to symmetry, only a quarter of a groove has to be considered. The mesh is denser in the AWZ and its vicinity and coarser far away, where important changes are less likely to occur, as it is shown in figure 14 (c). The details of the numerical routine can be found in reference (Osorio et al., 2008). We just indicate here the main steps of our calculations. 


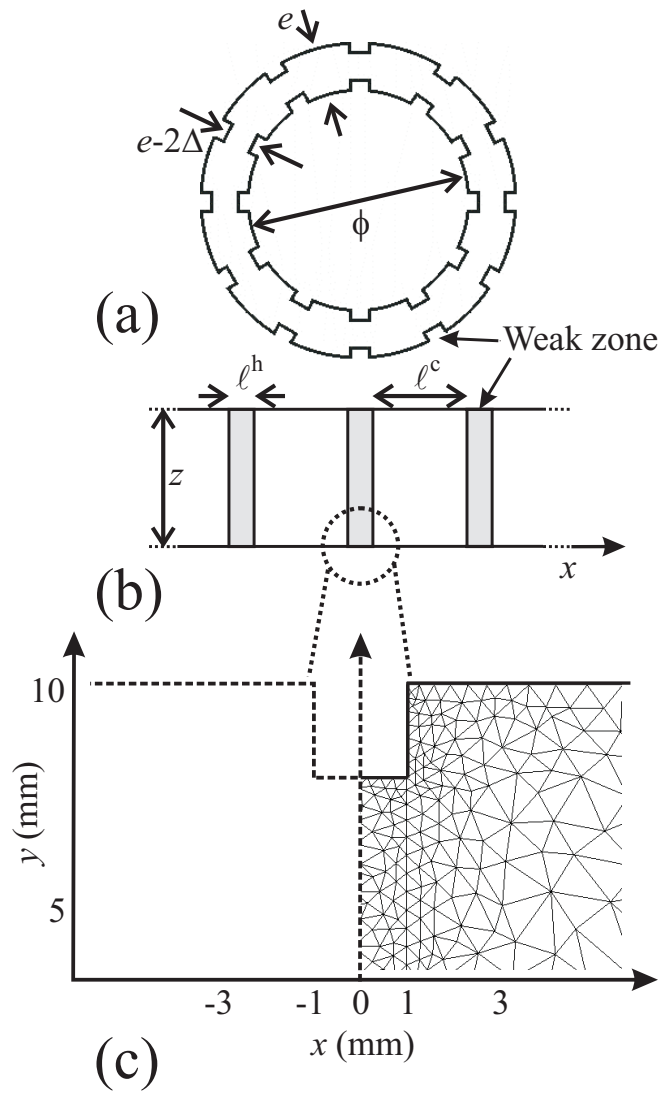

Fig. 14. (a) Schematic top view of the grooved cylinder under study. (b) Lateral view of a segment of the cylinder's perimeter used in the simulation. (c) Top view of the mesh used in FEM calculations. Due to symmetry, only a quarter of a groove is used. Figure from Ref.

(Osorio et al., 2008).

When the fault occurs, the circulating current overtakes the critical value $I_{\mathcal{C}}$ and dissipation arises. Following the temperature and current dependent equations for the electric field, the dissipation is calculated and so the temperature at each node of the mesh. Heat exchanges by convection with the coolant and conduction between hot and cold segments are modeled by taking into account experimental values for the temperature dependent convective coefficient for liquid nitrogen (Duron et al., 2007) and helium gas (Chapman, 1984), and the thermal conductivity (Fujishiro et al., 2003; 2006). As we are mainly interested on the thermal recovery, the condition that the circulating current is constant (not realistic for an inductive SFCL) does not preclude at all the validity of our conclusions about the recovery time. More sophisticated models, where the current changes according to the resistance developed in the superconducting element can be found elsewhere (Rettelbach \& Schmitz, 2003).

Figure 15 displays the temperature distribution within the cylinder's wall after a fault of 100 $\mathrm{ms}$. We can see that, despite the high temperature attained in the inner of the wall, the heating 


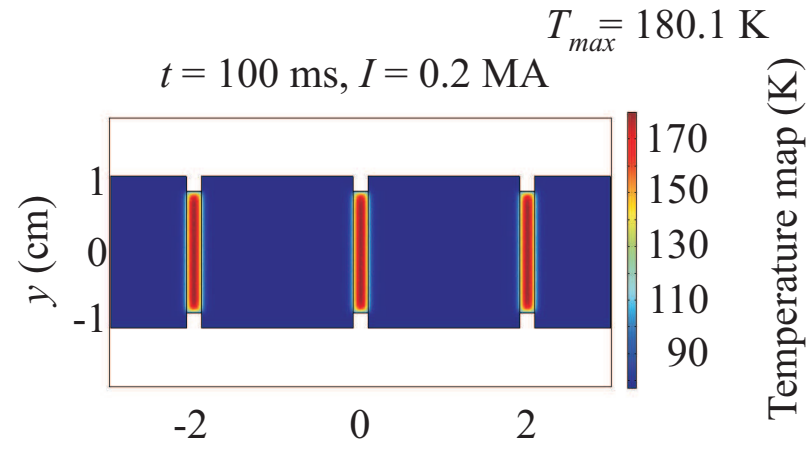

Fig. 15. Temperature map of a superconducting element after a fault of $100 \mathrm{~ms}$ in duration. Figure from Ref. (Osorio et al., 2008).

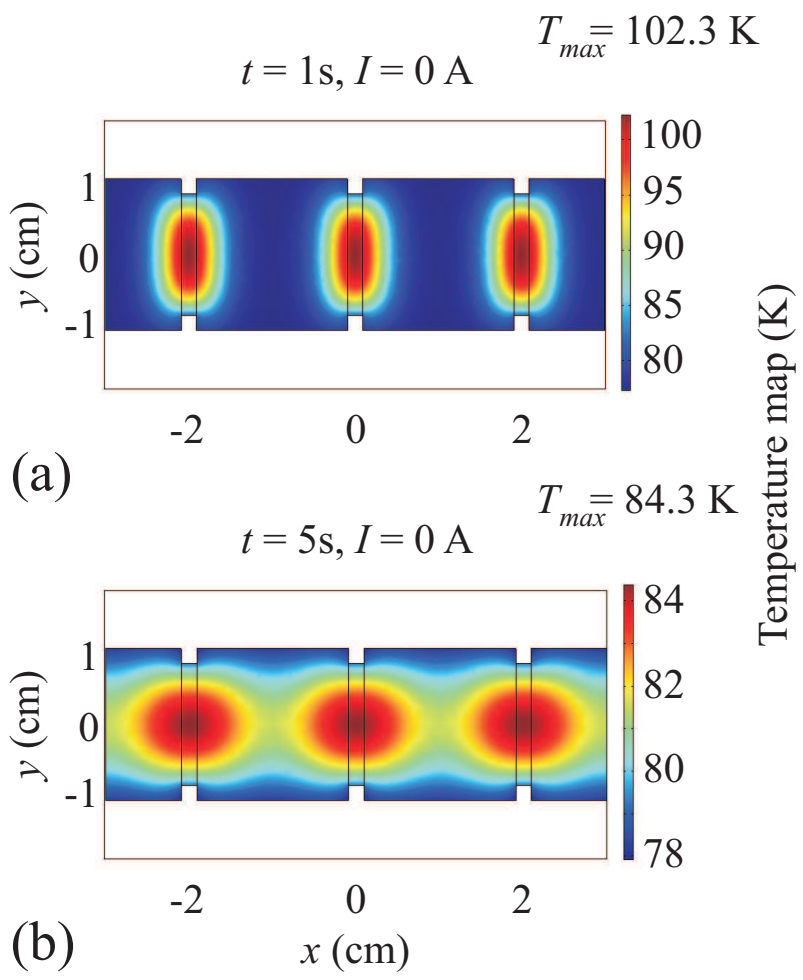

Fig. 16. Thermal recovery for the example of Figure 15 after $1 \mathrm{~s}$ of zero-current recovery (a) and after $5 \mathrm{~s}$ (b). Figure from Ref. (Osorio et al., 2008).

is quite confined to the AWZ. This effect of "non-propagation" of the hot spot during the fault was one of the main assumptions of the 1-D model, and this more refined model confirms its validity. The evolution of the hot spot once the fault is removed and the current is set to zero is shown in figures 16(a) and 16(b) for $t=1 \mathrm{~s}$ and $t=5 \mathrm{~s}$, respectively. Notice that the 
redistribution of the excess heat, confined at the beginning in the close vicinity of the weak zone, leads to a temperature map which is not so far above $77 \mathrm{~K}$.

Figure 17 shows an example of the variation of the primary current before and during a fault when the secondary is an ungrooved cylinder (i.e. without AWZ) and a grooved one, UC and GC1, respectively. Both samples are supposed to be made from YBCO, and GC1 is characterized by a ratio of grooved to ungrooved cross-sections $a=0.8$, and by the lengths of the hot and cold parts being $\ell^{h}=0.5$ and $\ell^{c}=0.5 \mathrm{~cm}$. Notice that the limited current is the same no matter the secondary used, so making grooves does not necessarily affect the limitation capacity.

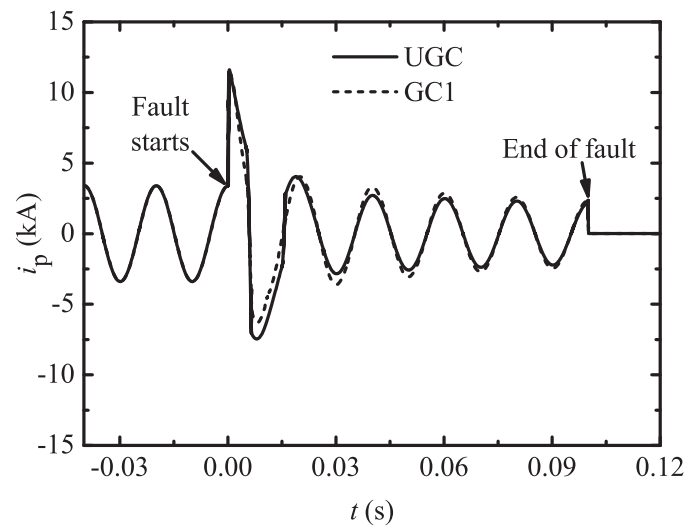

Fig. 17. Variation of the primary current for an ungrooved cylinder (UGC) and a grooved cylinder (GC1). Notice that the limitation performance is identical. Figure from Ref.

(Osorio et al., 2008).

As the temperature of the grooves can be very high and the thermal conductivity of YBCO within the $a-b$ planes is quite low, about $2.5 \mathrm{Wm}^{-1} \mathrm{~K}^{-1}$, it was proposed to use cylinders of YBCO grown in such a way that the current circulates along the $c$ axis (Osorio et al., 2008). This entails that the normal state resistivity is 15 times higher (Vanderbemden et al., 1999), and that the thermal conductivity along the perimeter is $10 \mathrm{Wm}^{-1} \mathrm{~K}^{-1}$ (and $3 \mathrm{Wm}^{-1} \mathrm{~K}^{-1}$ along the cross-section) (Fujishiro et al., 2003; 2006). With these new parameters, the AWZ can be made thinner without reducing the normal state resistance, while the removal of heat is made easier due to the higher thermal conductivity and to the reduction of the path the heat must travel to be exchanged between hot and cold parts. Figure 18 shows our best result for a grooved cylinder characterized by $a=0.8, \ell^{c}=0.8 \mathrm{~cm}$ and $\ell^{h}=0.2 \mathrm{~cm}$, when the limiter works in a liquid nitrogen bath (a) and a helium gas atmosphere (b). The thin dotted line shows the temperature at which the recovery ends. Notice that the recovery times for the grooved sample are about $1 \mathrm{~s}$, as demanded in practical applications. The solid lines correspond to the ungrooved cylinder. When a liquid nitrogen bath is used, the recovery for the ungrooved cylinder occurs at about $3.5 \mathrm{~s}$, despite the initial temperature is much lower. For the helium gas atmosphere, its temperature does not even change within the time interval under study (further details of these studies can be found in (Osorio et al., 2008)). 


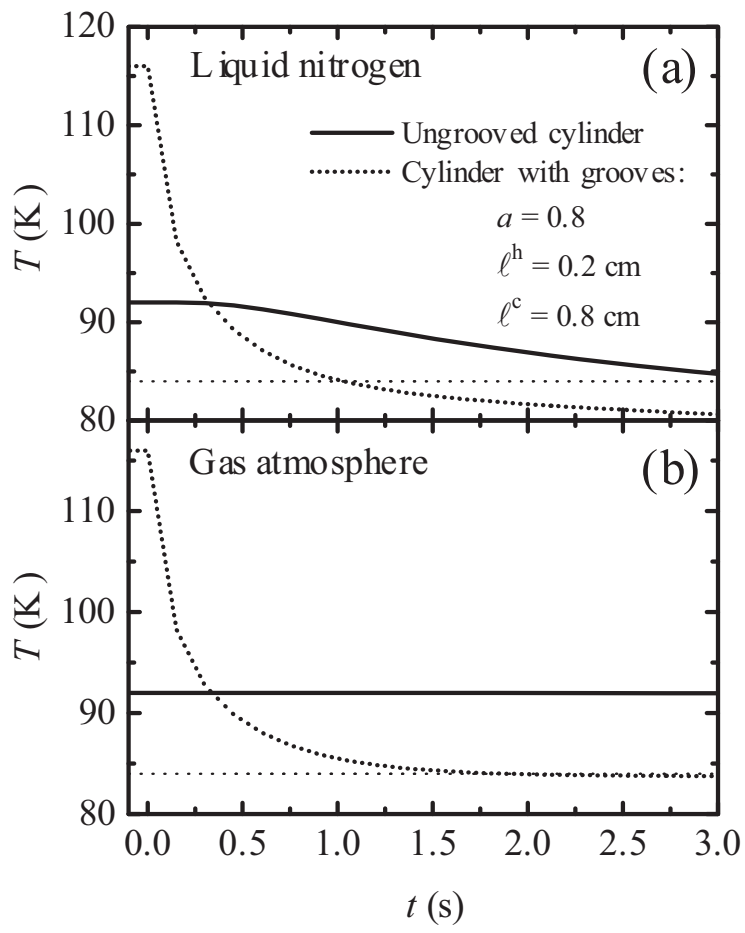

Fig. 18. Thermal recovery for an ungrooved cylinder (solid line) and for a sample with grooves characterized by $a=0.8, \ell^{c}=0.8 \mathrm{~cm}$ and $\ell^{h}=0.2 \mathrm{~cm}$ in liquid nitrogen (a) and helium gas (b). The thin dotted lines indicate the temperature at which no dissipation arises in the superconducting elements. Figure from Ref. (Osorio et al., 2008).

Our results show that the use of grooves samples can improve the performance of bulk superconductors working in SFCL. However such improvement requires a set of grooves with very close behaviour. We illustrate the importance of this requirement with the example displayed in Fig. 19. When all grooves are identical, which is the case discussed above, the temperature increase of these parts under current fault should reach only up to around $100 \mathrm{~K}$ (Fig. 19(a)). If one of the grooves presents a slightly greater ratio $a$ (around 1\%) the temperature map changes very little (Fig. 19(b)) and the main efect would be a small decrease of the fault impedance. One major drawback however occur if any of the grooves is smaller than the other: the temperature of the groove with $a$ around $1 \%$ smaller should increase up to around $150 \mathrm{~K}$ (Fig. 19(c)) which would cause a very long thermal recovery time after fault and therefore a poorer overall performance of the limiter device (a deeper study of this type of drawback can be found in (Osorio et al, 2010)).

\section{Stacks of bulk rings and thin film washers as secondary}

In the above section we have seen a way to improve the cooling of bulk superconducting samples by making a set of artificial weak zones. Another procedure could be increase the 


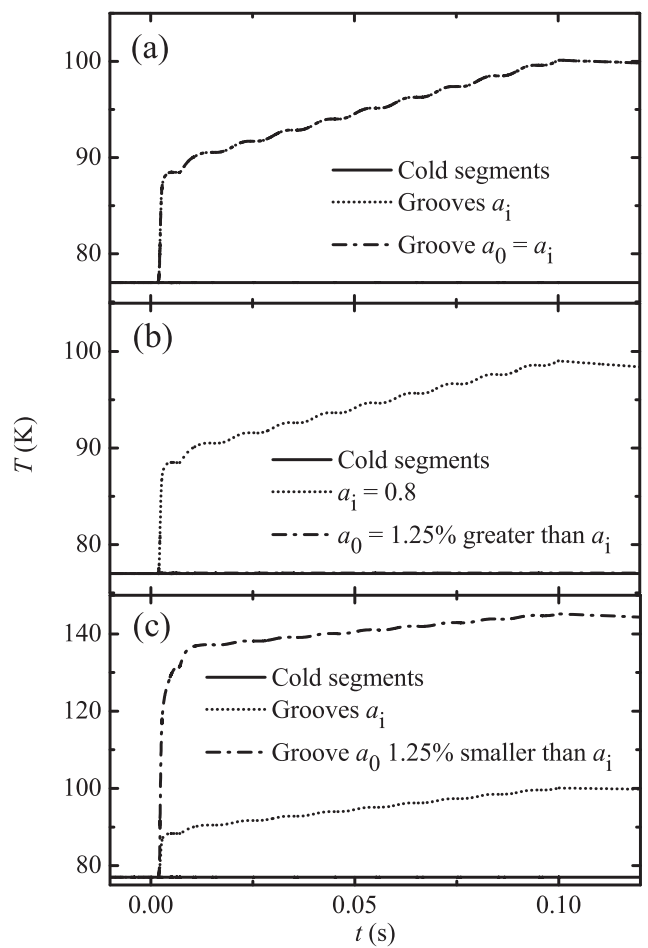

Fig. 19. Temperature excursions under current fault of cold parts and grooves for: (a) All of grooves with the same ratio $a$; (b) and (c) One of the grooves with greater and smaller $a$, respectively, than the other. Figure from Ref. (Osorio et al, 2010).

surface-to-volume ratio of the secondary by using short bulk rings or thin film washers. This idea was proposed in a previous work (Osorio et al., 2006), when it was presented a comparison between the performance of both types of secondaries. To balance the reduction in the effective critical current of the limiter (due to the lower cross-section of rings and washers when compared to bulk cylinders), it was proposed to stack these elements up to add up their critical current values.

In figure 20 it is displayed the impedance under current fault for three secondaries: a 40 mm-high Bi-2223 cylinder (labeled 1R40) a stack of three $2 \mathrm{~mm}$-high Bi-2223 rings (labeled $3 R 2$ ), and a stack of three $4.5 \mathrm{~mm}$-wide $\mathrm{Y} 123 / \mathrm{Au}$ washers (labeled 3W4.5). Y123/Au washers were trepanned from wafers made from a $\mathrm{Y}_{1} \mathrm{Ba}_{2} \mathrm{Cu}_{3} \mathrm{O}_{7-\delta}$ thin film ( $300 \mathrm{~nm}$ thick) shunted by a gold layer (100 nm thick), deposited on a $\mathrm{AlO}_{2}$ substrate provided by Theva (Germany).

It can be seen that these secondaries present a very different behaviour under a current fault; with $1 R 40$ the increase of impedance is very smooth, whereas it is somewhat abrupter with $3 R 2$ and much more with $3 W 4.5$. The differences are even more remarkable after the current fault. With $1 R 40$ the impedance for the highest voltage continues to increase. On the contrary, with $3 R 2$ the thermal recovery (i.e. when the circulating current is the same than before the 


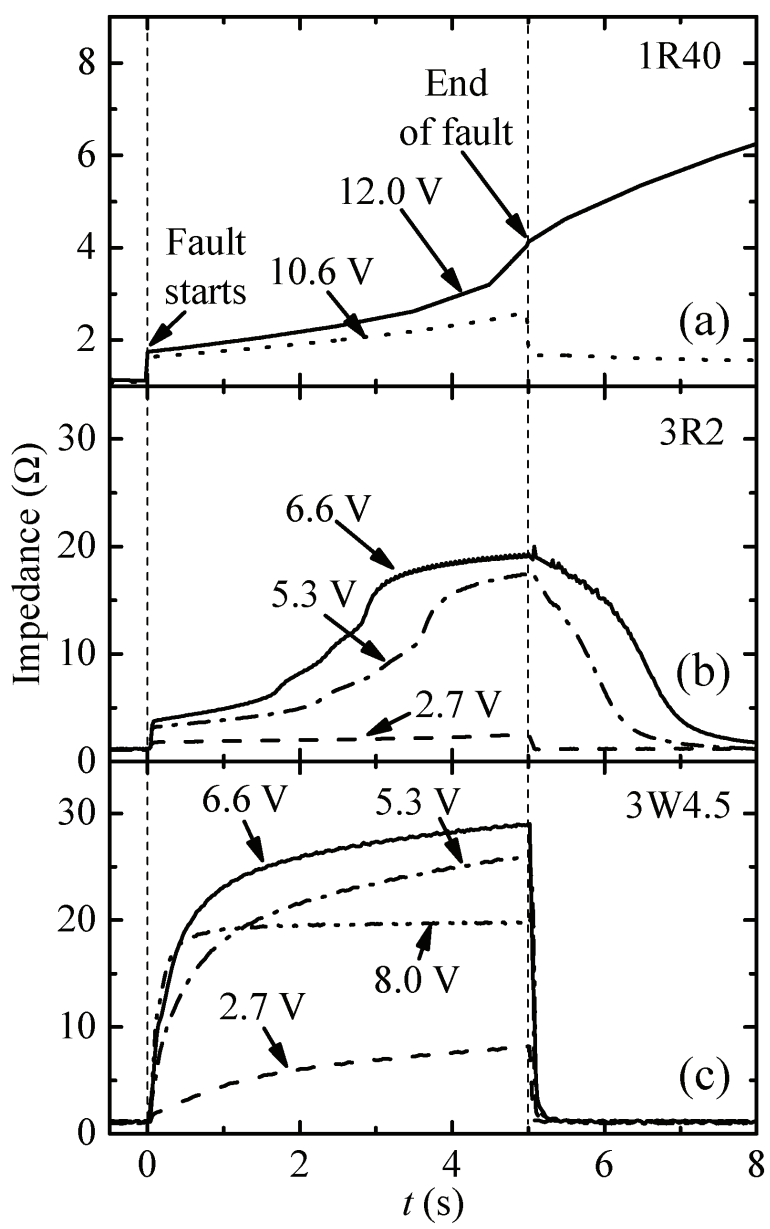

Fig. 20. Impedance at different fault voltages versus time for a limiter working with (a) 1 bulk Bi2223 ring of $40 \mathrm{~mm}$ in height, (b) a stack of 3 bulk Bi2223 rings of $2 \mathrm{~mm}$ in height and (c) a stack of 3 thin-film Y123/Au washers of $4.5 \mathrm{~mm}$ in width. Figure from Ref.

(Osorio et al., 2006).

fault) is accomplished within a few seconds, and it is very fast for $3 W 4.5$. The recovery times obtained for different faults and these three secondaries are plotted in figure 21 . For the secondaries $3 R 2$ and $3 W 4.5$ the recovery time values plotted were directly obtained from the measured current waveforms, assuming that the limiter was recovered when the amplitude of the current is the same as before the fault. The scattering of the data points around the guide lines, about $10 \%$, represents the precision of our measurements. With this procedure, the recovery times obtained could be somewhat overestimated because the circuit was not opened after each fault. Therefore some slight dissipation in the samples could still remain during the recovery process. Despite these small uncertainties, our results clearly show the better performance of the thiner samples. The secondaries $3 R 2$ and $3 W 4.5$ show recovery 


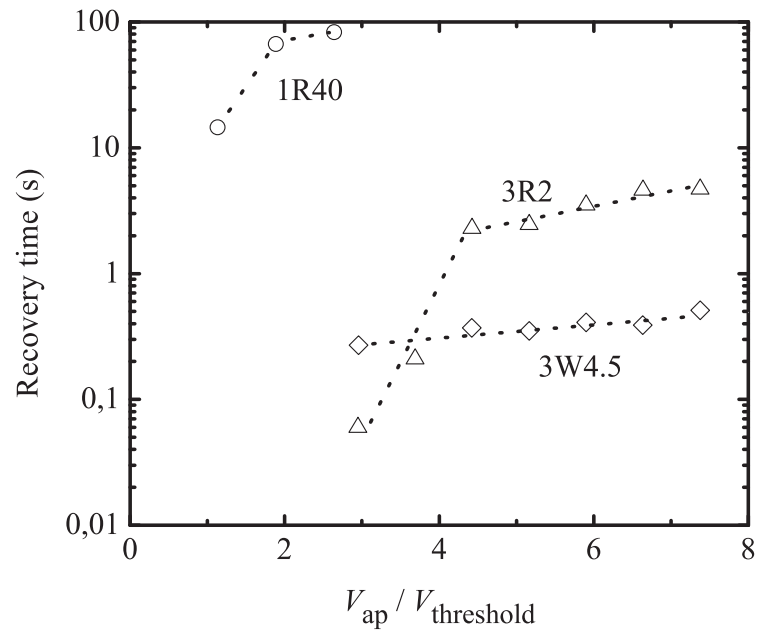

Fig. 21. Recovery time versus applied voltage normalized to the threshold value for the limiter working with a stack of three bulk Bi2223 rings of $2 \mathrm{~mm}$ in height (triangles) and a stack of three thin-film Y123/Au washers of $4.5 \mathrm{~mm}$ in width (diamonds). It is also included the recovery time when using as secondary a bulk Bi2223 ring of $40 \mathrm{~mm}$ in height (circles) $\left(V_{\text {threshold }}=7 \mathrm{~V}\right.$ for $1 R 40$ and $0.9 \mathrm{~V}$ for $3 R 2$ and $\left.3 W 4.5\right)$. Figure from Ref. (Osorio et al., 2006).

times one and, respectively, two orders of magnitude lower than the one for $1 R 40$. Note that the lowest times obtained for $3 R 2$ correspond to trivial examples of low fault voltages for which the samples develop very low resistance.

Furthermore, although $3 R 2$ and $3 W 4.5$ operate at a lower power rating than $1 R 40$, the recovery time will remain unchanged if more rings or washers were added to the stack. For instance, with about 25 of our thin films or short rings one should obtain the same operation currents than for the $1 R 40$ ring, the secondary being of similar size, with similar or higher impedance but with recovery time strongly shortened, specially with thin films. With this procedure it should be also possible to fabricate limiters able to operate at the high currents withstood, for instance, by many resistive type limiters based on meander thin films, which also present recovery time values in the range of tenths of seconds even when submerged in liquid nitrogen.

In case of a bulk cylinder (1R40) of the same diameter as the bulk rings and height $4 \mathrm{~cm}$, a low current (even below the nominal one) circulating after the fault is enough, as discussed above, to provoke appreciable dissipation in the sample. Therefore, the recovery time is extremely enlarged, even for the lowest applied voltages. So, for this secondary the recovery times were obtained by opening the circuit just after the end of the fault, i.e., we measure the zero current recovery time. With this procedure, the temperature at which the application of the nominal current does not generate dissipation is measured with the thermocouples attached on the rings. For 40-mm-high rings we found that the nominal current does not produce any appreciable heating for temperatures around $5 \mathrm{~K}$ above the working value $(77 \mathrm{~K})$. The circles plotted in figure 21 represent the interval between the end of the fault and the moment at 
which this temperature is reached. The precise value of the temperature threshold and the differences from sample to sample do not change the values of the recovery time in more than $20 \%$.

We must note that using stacks of bulk rings provokes a loss of impedance, as the superconducting elements of the stack would behave as resistances connected in parallel (Osorio et al., 2004). Although this drawback could be balanced by joining together several cores (Osorio et al., 2004), stacks of thin films show a better thermal performance and there is no reduction in the impedance or this is negligible (Lorenzo et al., 2006; 2007). Therefore, thin films offer important advantages concerning their refrigeration and the impedance does not diminish even when many of them are stacked to increase the effective critical current of the limiter.

\section{SFCL based on superconducting thin film microbridges and meander paths}

Thin film samples present several advantages to be used in current limiting applications, as we have discussed above. We have probed the use of this kind of samples in other two up to now nearly unexplored configurations. One is a hybrid limiting device (i.e inductive-resistive) refrigerated by using a thermoacoustic refrigerator. The other is the microlimiter, a resistive FCL based on "thermally small" (Ferro et al., 2008; 2009) superconducting microbridges (Lorenzo et al., 2009; 2010) intended to operate at very low powers (SQUID based electronics, infrared detectors, etc).

\subsection{Hybrid SFCL integrated in a thermoacoustic refrigerator}

Thermoacoustic refrigerators, heat engines which are based on the ability of a sound wave to play the role of the mechanical compressor and expander (Swift, 2002), have gained attention in the last decades and the state of art has allowed reaching very low temperatures (Yang \& Thummes, 2005; Qiu et al., 2005). Thermoacoustic devices are for the time being slightly less efficient than conventional refrigerators. Nevertheless, their simplicity, low cost and lack of moving parts (which entails a strong diminution of maintenance costs, the main trouble with commercial refrigerators) and contaminating lubricants offer advantages over certain other mechanical devices like mechanical compressors.

A scheme of a thermoacoustic refrigerator is shown in figure 22. It consists of a resonant tube filled with an inert gas (usually Helium at a high pressure, about tens of bars) in which a standing sound wave is generated by using an engine (which can be also of thermoacoustic nature, or just a compressor). Two heat exchangers are located at precise locations inside the tube and they are separated by a porous medium (regenerator) which acts as a heat pump, transferring heat from the cold heat exchanger to the ambient one (kept at room temperature by a water stream), by means of thermoacoustic processes (Swift, 2002). The proper phase between the acoustic pressure and velocity inside the regenerator (where a local traveling wave must exist to verify the heat pumping) is set by an acoustic charge, usually made up from a constriction, a long and thin tube and a huge cavity which behave as a resistor, an inductor and a capacitor in electrical circuits. A third heat exchanger (the "hot" one) is used to prevent the entrance of heat from the acoustic charge into the cold heat exchanger. When the engine is of thermoacoustic nature, the acoustic wave can be generated by a thermal gradient in an appropriately designed porous element and the heating source can be electric power 
(a)

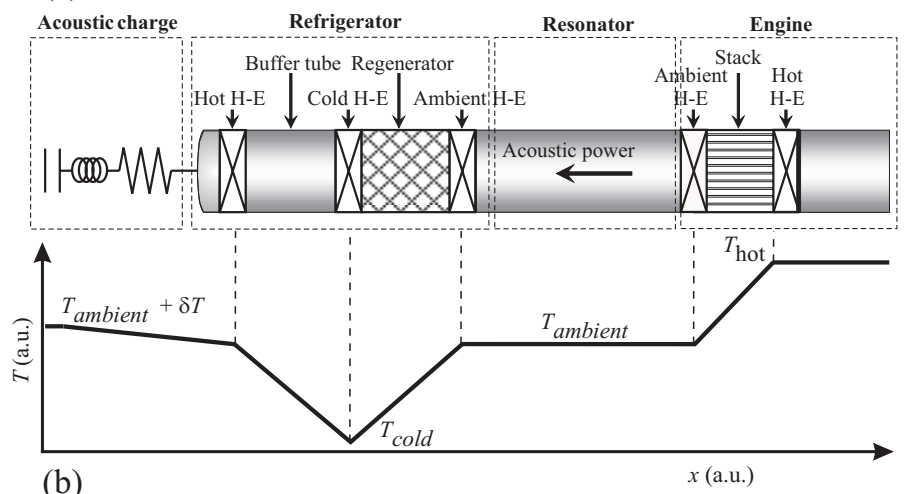

(b)

Fig. 22. (a) Schematic view of a thermoacoustic machine. On the right it can be seen the thermoacoustic engine. The thermoacoustic refrigerator, of pulse tube type, is comprised of three heat exchangers (HE), a regenerator and the buffer tube, which limits the entrance in the cold part of the heat generated by the dissipation of acoustic power in the charge. (b) Temperature profile along the TA machine. A strong gradient is imposed on the engine, so the wave can be generated. It is assumed that the dissipation increases the temperature in the acoustic charge. Figure from Ref. (Osorio et al., 2008).

(produced in a photovoltaic system, for instance) or burnt gas, as has already been done in some natural gas plants, where a conventional liquefying system were not cost effective.

In a previous work (Osorio et al., 2008) we proposed a $2.2 \mathrm{kVA}$ prototype of hybrid fault current limiter based on a YBCO film meander path which was directly attached to the cold heat exchanger of a thermoacoustic refrigerator, as it is schematically depicted in figure 23. The main advantage of this configuration is that the close contact between the superconducting path and the cold heat exchanger allows a very efficient removal of the excess heat produced during a fault, thus greatly improving the performance of refrigerators in which the element to be cooled is inside an isolated cavity connected to the cooler through some kind of pipe. The thermoacoustic refrigerator was intented to remove about $50 \mathrm{~W}$ at 80 $\mathrm{K}$ so the recovery time of the whole SFCL does not exceed $1 \mathrm{~s}$. The final design was tested by using a numerical routine (Osorio et al., 2008) and it was found that the SFCL was able to reduce the fault current in about 3 orders of magnitude, while the superconducting path heated about $130 \mathrm{~K}$ during a fault of $100 \mathrm{~ms}$ in duration. The total heat deposited in the cold heat exchanger was around $30 \mathrm{~J}$, low enough to be removed by the refrigerator in $1 \mathrm{~s}$.

Resistive configurations were not considered as the length of the superconducting path necessary to get a suitable impedance would be excessive to allow attaching it on the cold heat exchanger of a medium-size thermoacoustic refrigerator. In addition, all the fault power would be dissipated just in the meander path, and so the temperature increase could be very high. A hybrid configuration offers the advantages of a lower dissipation in the superconducting element (part of the energy is required to get the magnetic field inside the core) and also a high impedance which does not demand for a very long meander path. A pure inductive limiter was immediately rejected as the core would occupy too much room inside the refrigerator. 


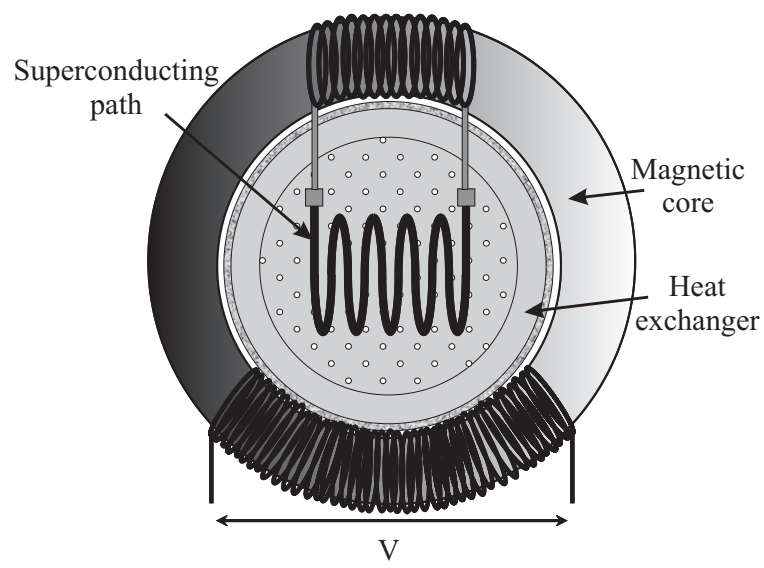

Fig. 23. Axial view of a hybrid limiter attached to the cold heat exchanger. The superconducting element is represented as a meander path. Figure from Ref. (Osorio et al., 2008).

\subsection{Superconducting fault current microlimiters}

In figure 24 we show a typical electric field versus current density $(E-J)$ curve, corresponding to one of the microbridges (denoted BS7) used in our experiments. This microbridge, whose length, width and thickness are, respectively, $385 \mu \mathrm{m}, 28 \mu \mathrm{m}$ and $300 \mathrm{~nm}$, and with $T_{c}=88.6$ $\mathrm{K}$, has been grown on a sapphire substrate. The two characteristic current densities are indicated in this figure: The critical, $J_{c}$, at which dissipation first appears, and the so-called supercritical, $J^{*}$, at which the microbridge is triggered into highly dissipative states. Let us note here that we have chosen for our studies microbridges of these dimensions to guarantee

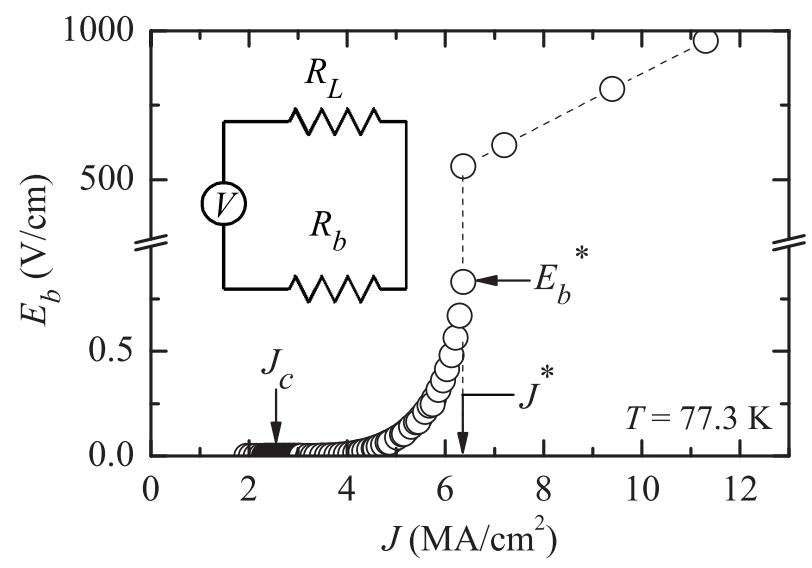

Fig. 24. A typical $E-J$ curve obtained at $T=77.3 \mathrm{~K}$ in one of the microbridges used in our experiments (BS7). The inset shows a schematic diagram of the circuit used to probe how the microbridge acts as a FCL, protecting the load resistance, $R_{L}$, from a voltage fault. Figure from Ref. (Lorenzo et al., 2009). 
a good thermal behaviour before, during and after the current fault, as we will see below. In addition, the widths of our microbridges are well above the threshold at which $J^{*}$ is sample-width dependent (Ruibal et al., 2007). The strong increase of $E$ for current densities around $J^{*}$ make this type of samples very useful for FCL. However, the current is effectively limited only for electric fields or, equivalently, applied voltages well above that at which the limiter is triggered from normal operation (sample in the superconducting state) to current fault mode. In addition, once $J^{*}$ is attained, a thermal runaway (Viña et al., 2003; Maza et al., 2008) can be provoked which causes the reduction of the circulating current well below the nominal value (i.e., the current in normal operation without a fault) or very important damage on the microbridge (that can be even burnt out).

The superconducting microbridge exchanges heat mainly with its substrate, because at the operation temperatures, around $90 \mathrm{~K}$, the heat transfer coefficient between YBCO films, for instance, and their substrate is $h_{b s} \approx 10^{3} \mathrm{Wcm}^{-2} \mathrm{~K}$, whereas through liquid nitrogen or between the substrate and the copper holder they are 1000 times less (Duron et al., 2007; Lorenzo et al., 2009; Mosqueira et al., 1993). Hence, the conditions for a good refrigeration and, therefore, an optimal operation of the limiting device, depend very much on the relative dimensions of the microbridge and their substrate. The optimal refrigeration should allow us to operate with the minimum microbridge temperature increment relative to that of the bath, $\Delta T_{b}$. If the Biot number (Lorenzo et al., 2009; Chapman, 1984) of both the microbridge and its substrate are $B i \ll 1$ and, simultaneously, the thermal diffusion length, $L_{t h}$, is longer than the corresponding thickness, $\Delta T_{b}$ may be crudely approached by

$$
\Delta T_{b}=e_{b} J E\left(\frac{1}{h_{b s}}+\frac{A_{b}}{A_{s}} \frac{1}{h_{s r}}\right),
$$

where $e_{b}$ is the microbridge thickness, $A_{b}$ and $A_{s}$ are the surface areas of the superconductor bridge and, respectively, its substrate. As $h_{b s} \gg h_{s r}, \Delta T_{b}$ will directly depend on $A_{b} / A_{s}$, the condition of "thermal smallness" being,

$$
\frac{A_{b}}{A_{s}}<\frac{h_{s r}}{h_{b s}}
$$

under which $\Delta T_{b}$ approaches its "intrinsic" value, which is the temperature increase just associated with the effective thermal resistance of the interface bridge-substrate. For our microbridges similar to BS7 and under faults with characteristic times above $10 \mathrm{~ms}$ (of the order of commercial ac current periods), the conditions, $B_{i} \ll 1$ and $L_{t h}>e$ fully apply. In addition, as $A_{b} / A_{s} \approx 4 \times 10^{-4}$ whereas $h_{s r} / h_{b s} \approx 10^{-3}$, this microbridge is "thermally small". One may use equation 5 to estimate $\Delta T_{b}$ when the microbridge is in stationary conditions under currents just below $J^{*}$. From figure 24 , the power density involved is around $5 \times 10^{6}$ $\mathrm{W} / \mathrm{cm}^{3}$ which leads, by also using the appropriate parameter values ${ }^{1}$, to $\Delta T_{b} \approx 0.1 \mathrm{~K}$. Even for faults involving powers ten times higher than those considered above, $\Delta T_{b}$ will remain below $1 \mathrm{~K}$. One may also use equation 5 to roughly estimate that if the superconducting bridge had $A_{b} / A_{s}$ one thousand times larger, as it is the case of those currently proposed for high power applications (Noe \& Steurer, 2007; Weinstock, 2000), $\Delta T_{b}$ would take values at least

\footnotetext{
1 The substrate thermal conductivities $\left(K_{s}\right)$ and heat capacities $\left(C_{s}\right)$ are of the order of $10 \mathrm{~W} / \mathrm{cm} \mathrm{K}$ and 0.6 $\mathrm{J} / \mathrm{cm}^{3} \mathrm{~K}$ for sapphire, and $0.2 \mathrm{~W} / \mathrm{cm} \mathrm{K}$ and $1 \mathrm{~J} / \mathrm{cm}^{3} \mathrm{~K}$ for $\mathrm{SrTiO}_{3}$, whereas for $\mathrm{YBCO}$ bridges $K_{b} \approx 0.05$ $\mathrm{W} / \mathrm{cm} \mathrm{K}$ and $C_{b} \approx 0.7 \mathrm{~J} / \mathrm{cm}^{3}$ K. See, e. g., Refs. (Duron et al., 2007; Chapman, 1984; Mosqueira et al., 1993).
} 
two orders of magnitude higher. To confirm these values at a quantitative level, $\Delta T_{b}$ has been calculated by using a finite element method similar to the one described in (Maza et al., 2008). Under the same conditions as above, for the BS7 microbridge we found again $\Delta T_{b} \approx 0.1 \mathrm{~K}$.

In the case of $\mathrm{SrTiO}_{3}$ substrates, which have relatively poor thermal conductivities, the condition $B i \ll 1$ does not apply anymore. Therefore, a term proportional to $1 / k_{s}$ (i.e. to the thermal conductivity of the substrate) must be added in equation 5 . For microbridges under the same conditions as before, this leads to $\Delta T_{b} \approx 2 \mathrm{~K}$, a value that is confirmed by using the finite element method commented above. Let us stress, finally, that for a bridge on sapphire but having a surface relative to that of its substrate one thousand times larger, the finite element method yields $\Delta T_{b} \approx 50 \mathrm{~K}$.

To probe a microlimiter with low thermal dimensions, we have implemented the electrical circuit schematized in the inset of figure 24 , with the microbridge BS7 as $R_{b}$ connected in series to the variable load resistance $R_{L}$, this last one representing the impedance of the circuit to be protected. The measurements were made in a cryostat with the sample submerged in a forced flow of helium gas. The temperature of the copper holder of the microlimiter was measured with a platinum thermometer and regulated with an electronic system which ensures a temperature stabilization better than $0.05 \mathrm{~K}$. Two examples of the $I-V$ curves obtained in this $R_{L}-R_{b}$ circuit (with $R_{L}=4.9 \Omega$, this value taking already into account the resistance of the circuit electrical wires, of the order of $1.9 \Omega$ ) by using the electronic system described elsewhere (Ruibal et al., 2007; Viña et al., 2003) are shown in figure 25. In these curves the voltage was imposed and acquired during pulses of $1 \mathrm{~s}$, a time much longer than the one needed by the microlimiter to reach the stationary state. The bath temperatures were $81.9 \mathrm{~K}$ (circles) and $85.0 \mathrm{~K}$ (triangles). As below $V^{*}$ the flux-flow resistance of the microbridge remains much lower than $R_{L}$, both curves are almost linear up to $V^{*}$. Together with the low heating estimated above, this quasi-ohmic behavior is crucial to allow the microlimiter to work just below $V^{*}$ under stationary conditions and without disturbing the circuit to be protected.

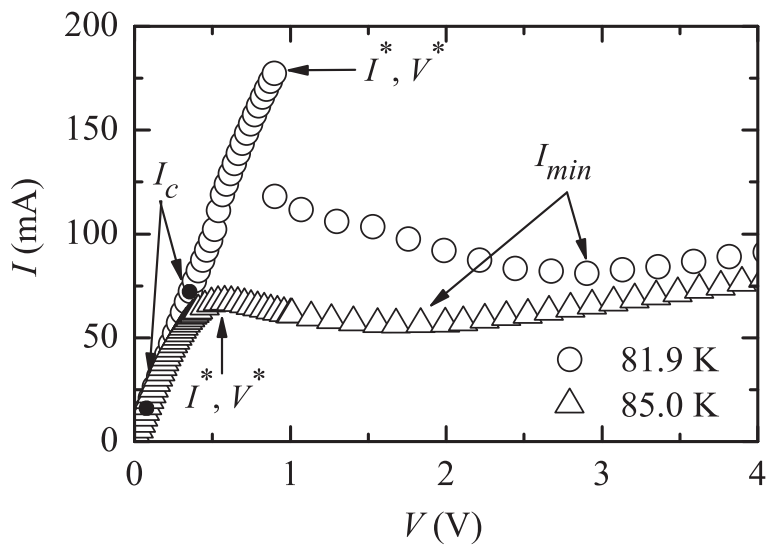

Fig. 25. Two $I-V$ curves, showing their strong bath temperature dependence, of the $R_{L}-R_{b}$ circuit schematized in the inset of figure 25 In these examples $R_{b}$ is the microbridge BS7 and $R_{L}=4.9 \Omega$. Figure from Ref. (Lorenzo et al., 2009). 


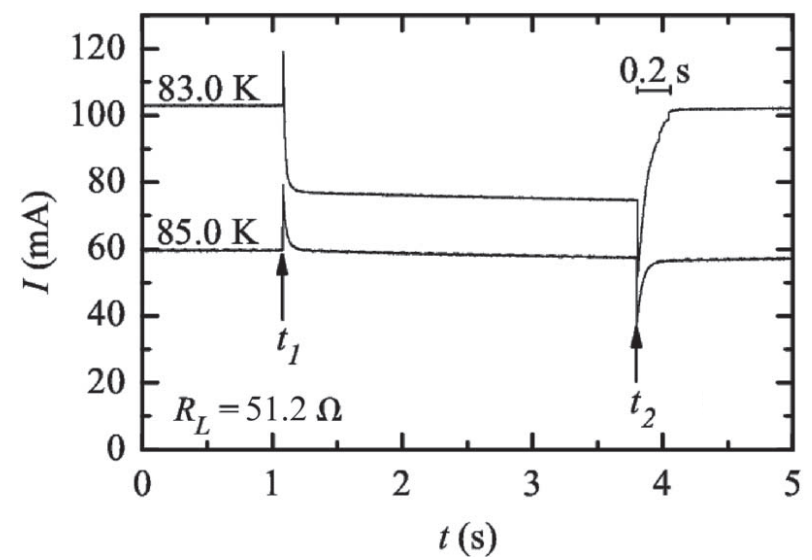

Fig. 26. Two examples of the time evolution of the current measured in presence of voltage faults between $t_{1}$ and $t_{2}$. Figure from Ref. (Lorenzo et al., 2009).

The results presented in figure 25 also illustrate two other central aspects of the microbridges when working as FCL. Note first that, once the source voltage overcomes $V^{*}$, the current in the circuit varies quite slowly even up to voltage faults as important as four times $V^{*}$, the current taking a minimum value, $I_{\text {min }}$, at some (temperature-dependent) voltage. Moreover, the sharp drop at $V^{*}$ of the current is also temperature dependent, being almost absent in the curve at $85.0 \mathrm{~K}$. Both aspects are related and may be explained in terms of the approaches based on the propagation of self-heating hot spots (Skocpol et al., 1974; Gurevich \& Mints, 1987; Paulin et al., 1995): Above $V^{*}$ part of the microbridge becomes normal and then, as the total voltage of the circuit is fixed, the resistance increase originates a current decrease up to the minimum current, $I_{\min }$, capable of sustaining the normal zone. If the fault voltage increases, the hotspot length will grow accordingly, keeping the current roughly constant. At a quantitative level, both aspects may be easily explained by just taking into account the reduced temperature $\left(T / T_{\mathcal{C}}\right)$ dependence of $I^{*}$ and $I_{\text {min }}$

$$
I(T)=I(0)\left(1-\frac{T}{T_{c}}\right)^{n},
$$

with $n=3 / 2$ for $I^{*}$ (Ruibal et al., 2007; Viña et al., 2003) and 1/2 for $I_{\text {min }}$ (Skocpol et al., 1974; Gurevich \& Mints, 1987; Paulin et al., 1995). Therefore, if the reduced temperature increases both the discontinuity at $V^{*}$ and the ratio $I^{*} / I_{\min }$ will decrease, in agreement with the results of figure 25 The "optimal" reduced temperature for the microlimiter operation, $T_{o p} / T_{c}$, will be then given by the condition $I^{*}\left(T_{o p}\right)=I_{\min }\left(T_{o p}\right)$. By using equation 7 , this leads to,

$$
\frac{T_{o p}}{T_{c}}=1-\frac{I_{\min }(0)}{I^{*}(0)} .
$$

At $T_{o p}$ the current limited during the voltage fault will be roughly equal to the nominal one. As $I_{\text {min }}(0)<I^{*}(0), T_{o p}$ will be near, but below enough, $T_{\mathcal{C}}$ to make $I^{*}\left(T_{o p}\right)$ adequate for the practical operation of the microlimiter under such an optimal temperature.

The above results, experimentally confirmed with our studies (Lorenzo et al., 2009; 2010), allow us to "thermally tune" a superconducting microlimiter for an optimal protection. The 
results summarized in figure 26 were measured by using a circuit similar to that of the inset of figure 24. In these examples, $R_{S}$ is again the BS7 microbridge and $R_{L}=51.2 \Omega$ (taking into account the resistance of the circuit electrical wires). For the curve measured at $83.0 \mathrm{~K}$, the applied voltage was $5.8 \mathrm{~V}$ before $\left(t_{1}\right)$ and after $\left(t_{2}\right)$ a fault regime of $9.5 \mathrm{~V}$. For the curve at $85.0 \mathrm{~K}$, which is near $T_{o p}$, these values were $3.5 \mathrm{~V}$ and $7.5 \mathrm{~V}$, respectively. As expected, the protection is excellent, whereas there is an overprotection when working well below $T_{o p}$. In both cases the recovery after the fault is achieved under current. This is another considerable practical advantage when compared to the superconducting limiters used in high power applications (Noe \& Steurer, 2007; Weinstock, 2000). These results suggest that optimal current limitation in superconducting electronics could be accomplished by the own conductive pathways after a proper design (e. g. by decreasing the width of the pathway at well refrigerated selected locations), thus improving compactness.

\section{Conclusion}

We have summarized some of our works on the thermal behaviour and the refrigeration of fault current limiters based on HTSC. Our results were experimentally or numerically obtained for the three basic SFCL configurations (resistive, inductive and hybrid types). For inductive devices we report on the effects on the limiter's performance of the inhomogeneous nature of HTSC, on the advantages of using this type of samples with artificially created weak zones or with stacks of elements of great surface-to-volume ratio, in particular thin film washers, which present a extremely large value of this ratio. The good refrigeration conditions of the superconducting elements obtained with these designs allow us to operate with limiters of improved thermal stability under a current fault and shorter recovery times after the fault removal. For bulk cylinders with AWZ, the recovery time values are several times lower than with homogeneous cylinders, while the impedance remains unchanged. By using stacks of bulk rings, with a large surface-volume ratio, the recovery time is about one order of magnitude shorter and without impedance loss. By using stacks of thin film washers, the recovery time is two orders of magnitude lower than with bulk cylinders and the impedance is even better.

For hybrid and resistive configurations, we have presented results for designs scarcely explored. On one hand, a limiter based on a thin film meander path integrated in the cold heat exchanger of a thermoacoustic refrigerator. The hybrid design of this device would allow us to operate at a power about $2.2 \mathrm{kVA}$ with a thermoacoustic refrigerator capable of removing about $50 \mathrm{~W}$ at $80 \mathrm{~K}$. Finally, we have probed the use of thin film microbridges to operate in very low power applications. Our results with these samples indicate that, by using the appropriate substrates and the right ratio microbridge-to-substrate area, it is possible to operate in a regime where a relative small fault provokes their transition to highly dissipative states that strongly increase the limitation efficiency.

\section{Acknowledgements}

The earlier phases of the research summarized here were partially supported by the electrical company UNION-FENOSA under Grants No. 0666-98 and 0220-0085. This work has been supported by Ministerio de Ciencia e Innovación ERDF FI2010-19807 and by Xunta de Galicia ERDF 2010/XA043 and 10TMT206012PR. 


\section{References}

Noe, M. \& Steurer, M. (2007). High-temperature superconductor fault current limiters: concepts, applications, and development status. Superconductor Science and Technology, Vol. 20, No. 3, January 2007, R15-R29, ISSN 0953-2048.

Sokolovsky, V.; Meerovich, V.; Vajda, I.; Beilin, V. (2004). Superconducting FCL: Design and Application. IEEE Transactions on Applied Superconductivity, Vol. 14, No. 3, September 2004, 1990-2000, ISSN 1051-8223.

Paul, W.; Chen, M.; Lakner, M.; Rhyner, J.; Braun, D.; Lanz, W. (2001). Fault current limiter based on high temperature superconductors - different concepts, test results, simulations, applications. Physica C, Vol. 354, No. 1-4, May 2001, 27-33, ISSN 0921-4534.

Paul, W.; Lakner, M.; Rhyner, J.; Unternährer, P.; Baumann, Th.; Chen, M.; Widenhorn, L.; Guérig, A. (1997). Test of 1.2 MVA high-Tc superconducting fault current limiter. Superconductor Science and Technology, Vol. 10, No. 12, December 1997, 914-918, ISSN 0953-2048.

Leung, E.M.; Rodriguez, I.; Albert, G.W.; Burley, B.; De, M.; Gurrola, P.: Madura, D.; Miyata, G.; Muehleman, K.; Nguyen, L.; Pidcoe, S.; Ahmed, S.; Dishaw, G.; Nieto, C.; Kersenbaum, I.; Gamble, B.; Russo, C.; Boenig, H.; Peterson, D.; Motowildo, L.; Haldar, P. (1997). High temperature superconducting fault current limiter development. IEEE Transactions on Applied Superconductivity, Vol. 7, No. 2, June 1997, 985-988, ISSN 1051-8223.

Berends, F.A.; Papadopoulos, C.G.; Pittau, R.; Zueger, H. (1998). 630 kVA high temperature superconducting transformer. Cryogenics, Vol. 38, No. 11, November 1998, 1169-1172, ISSN 0011-2275.

Antognazza, L.; Decroux, M.; Reymond, S.; de Chambrier, E.; Triscone, J.M.; Paul, W.; Chen, M.; Fischer, Ø. (2002). Simulation of the behaviour of superconducting YBCO lines at high current densities. Physica C, Vol. 372-376, No. 3, August 2002, 1684-16877, ISSN 0921-4534.

Osorio, M.R.; Lorenzo, J.A.; Toimil, P.; Ferro, G.; Veira, J.A.; Vidal, F. (2006). Inductive superconducting fault current limiters with Y123 thin-film washers versus Bi2223 bulk rings as secondaries. IEEE Transactions on Applied Superconductivity, Vol. 16, No. 3, September 2006, 1937-1942, ISSN 1051-8223.

Decroux, M.; Antognazza, L.; Reymond, S.; Paul, W.; Chen, M.; Fischer, Ø. (2003). Studies of YBCO strip lines under voltage pulses: optimization of the design of fault current limiters. IEEE Transactions on Applied Superconductivity, Vol. 13, No. 2, June 2003, 1988-1991, ISSN 1051-8223.

Antognazza, L.; Decroux, M.; Therasse, M.; M. Abplanalp, M.; Fischer, Ø. (2005). Test of YBCO thin films based fault current limiters with a newly designed meander. IEEE Transactions on Applied Superconductivity, Vol. 15, No. 2, June 2005, 1990-1993, ISSN 1051-8223.

Duron, J.; Antognazza, L.; Decroux, M,; Grilli, F.; Stavrev, S.; Dutoit, B.; Fischer, Ø. (2005). 3-D finite element simulations of strip lines in a $\mathrm{YBCO} / \mathrm{Au}$ fault current limiter. IEEE Transactions on Applied Superconductivity, Vol. 15, No. 2, June 2005, 1998-2002, ISSN 1051-8223.

Duron, J.; Dutoit, B.; Grilli, F.; Decroux, M.; Antognazza, L.; Fischer, Ø. (2007). Computer Modeling of YBCO Fault Current Limiter Strips Lines in Over-Critical Regime With 
Temperature Dependent Parameters. IEEE Transactions on Applied Superconductivity, Vol. 17, No. 2, June 2007, 1839-1842, ISSN 1051-8223.

Antognazza, L.; Decroux, M.; Therasse, M.; Abplanalp, M.; Duron, J.; B. Dutoit, B.; Fischer, Ø. (2007). Thermally Assisted Transition in Thin Film Based FCL: A Way to Speed Up the Normal Transition Across the Wafer. IEEE Transactions on Applied Superconductivity, Vol. 17, No. 2, June 2007, 3463-3466, ISSN 1051-8223.

Duron, J.; F. Grilli, F.; L. Antognazza, L.; M. Decroux, M.; B. Dutoit B.; Fischer, Ø. (2007). Finite-element modelling of YBCO fault current limiter with temperature dependent parameters. Superconductor Science and Technology, Vol. 20, No. 4, April 2007, 338-344, ISSN 0953-2048.

Osorio, M. R.; Ruibal, M.; Veira, J.A.; Vidal, F. (2005). Thermal recovery of inductive superconducting fault current limiters with weak zones in the secondary. Superconductor Science and Technology, Vol. 18, No. 5, April 2005, 739-746, ISSN 0953-2048.

Osorio, M. R.; Lorenzo Fernández, J. A.; Veira, J. A.; Vidal, F. (2008). Optimal refrigeration of bulk superconducting elements in fault current limiters by using artificial weak zones. Superconductor Science and Technology, Vol. 21, No. 9, July 2008, 095011(8pp), ISSN 0953-2048.

Osorio, M. R.; Cabo, L.; Veira J. A.; Vidal, F. (2004). Inductive fault current limiter based on multiple superconducting rings of small diameter. Superconductor Science and Technology, Vol. 17, No. 1, November 2003, 98-102, ISSN 0953-2048.

Osorio, M. R.; Bétrancourt, A.; François, M. X.; Veira, J. A.; Vidal, F. (2008). A superconducting fault current limiter integrated in the cold heat exchanger of a thermoacoustic refrigerator. Superconductor Science and Technology, Vol. 21, No. 9, July 2008, 095013(7pp), ISSN 0953-2048.

Ferro, G.; Ruibal, M.; Veira J. A.; Maza, J.; Vidal, F. (2008). Thermal effects in the flux-creep regime of $\mathrm{YBa}_{2} \mathrm{Cu}_{3} \mathrm{O}_{7-\delta}$ thin film microbridges under high current densities in self field. Journal of Physics: Conference Series, Vol. 97, No. 1, March 2008, 012016(5pp), ISSN 1742-6596.

Ferro, G.; Maza, J.; Ramallo, M.V.; Veira J. A.; Vidal, F. (2009). Influence of thermal, morphological and measuring variables on the thermal runaway of superconducting thin films under high current densities. Journal of Physics: Conference Series, Vol. 150, No. 5, March 2009, 052053(5pp), ISSN 1742-6596.

Lorenzo, J. A.; Osorio, M. R.; Veira J. A.; Vidal, F. (2009). High-temperature superconducting fault current microlimiters. Superconductor Science and Technology, Vol. 22, No. 2, January 2009, 025009(4pp), ISSN 0953-2048.

Lorenzo Fernández, J. A.; Ferro, G.; Osorio, M. R.; Veira J. A.; Tello, M.J.; Vidal, F. (2010). Thermal behaviour of high-temperature superconducting fault current limiters: Application to microlimiters. Journal of Physics: Conference Series, Vol. 234, No. 4, July 2010, 042019(6pp), ISSN 1742-6596.

Osorio, M. R.; Veira, J. A.; Vidal, F. (2010). Behaviour of different artificial weak zones in superconducting elements working in inductive fault current limiters. Journal of Physics: Conference Series, Vol. 234, No. 3, June 2010, 032045(7pp), ISSN 1742-6596.

Prester, M. (1998). Current transfer and initial dissipation in high-Tc superconductors. Superconductor Science and Technology, Vol. 11, No. , November 1998, 333-357, ISSN 0953-2048. 
Osorio, M. R.; Cabo, L.; Veira, J. A.; Vidal, F. (2004). Non-homogeneous quench of the superconducting secondary of an inductive fault current limiter: implications for current limitation. Superconductor Science and Technology, Vol. 17, No. 7, May 2004, 868-875, ISSN 0953-2048.

M.I.T. Staff. (1961). Magnetic Circuits and Transformers, John Wiley \& Sons, ISBN 10-0-262-63063-4, New York.

Paul, W.; Baumann, T.; Rhyner, J.; Platter, F. (1995). Tests of 100 kW High-Tc superconducting fault current limiter. IEEE Transactions on Applied Superconductivity, Vol. 5, No. 2, June 1995, 1059-1062, ISSN 1051-8223.

Xiao, L. Y.; Kiyoshi, T.; Ozaki, O.; Wada, H. (1999). Case Study on the Quench Evolution and Passive Protection of High Tc Superconducting Pancake Coil. Cryogenics, Vol. 39, No. 4, Paril 1999, 293-298, ISSN 0011-2275.

Davidson, \& M. Tinkham, A. (1976). Phenomenological equations for the electrical conductivity of microscopically inhomogeneous materials. Physical Review B, Vol. 13, No. 1, April 1976, 3261-3267, ISSN 0163-1829.

Osorio, M. R.; Toimil, P.; Lorenzo, J. A.; Ruibal, M.; Ferro, G.; Veira, J. A.; Vidal, F.; (2006). Quench and thermal recovery in a superconducting fault current limiter with artificial weak zones. Journal of Physics: Conference Series, Vol. 43, No. 1, June 2006, 929-932, , ISSN 1742-6596.

Chapman, A. J. (1984). Heat Transfer, MacMillan Publishing Company, ISBN 0-62-946080-8, New York.

Fujishiro, H.; Teshima, H.; Ikebe, M.; Noto, K. (2003). Thermal conductivity of YBaCuO bulk superconductors under applied field: effect of content and size of Y211 phase. Physica C, Vol. 392-396, No. 1, October 2003, 171-174, ISSN 0921-4534.

Fujishiro, H.; Ikebe, M.; Teshima, H.; Hirano, H. (2006). Low-Thermal-Conductive DyBaCuO Bulk Superconductor for Current Lead Application. IEEE Transactions on Applied Superconductivity, Vol. 16, No. 2, June 2006, 1007-1010, ISSN 1051-8223.

Rettelbach T. \& Schmitz, G. J. (2003). 3D simulation of temperature, electric field and current density evolution in superconducting components. Superconductor Science and Technology, Vol. 16, No. 5, May 2003, 645-653, ISSN 0953-2048.

Vanderbemden, Ph.; Cloots, R.; Ausloos, M.; Doyle, R. A.; Bradley, A. D.; Lo, W.; Cardwell, D. A.; Campbell, A. M.; (1999). Intragranular and intergranular superconducting properties of bulk melt-textured YBCO. IEEE Transactions on Applied Superconductivity, Vol. 9, No. 2, 2308-2311, ISSN 1051-8223.

Lorenzo Fernández, J. A.; Osorio, M. R.; Toimil, P.; Ferro, G.; Blanch, M.; Veira J. A.; Vidal, F. (2006). The impedance of inductive superconducting fault current limiters operating with stacks of thin film Y123/Au washers or bulk Bi2223 rings as secondaries. Superconductor Science and Technology, Vol. 19, No. 12, December 2006, 1274-1277, ISSN 0953-2048.

Lorenzo Fernández, J. A.; Osorio, M. R.; Toimil, P.; Ferro, G.; Blanch, M.; Veira, J. A.; Tello, M.; Vidal, F. (2007). Improvement of an inductive fault current limiter with the use of stacks of thin film washers as secondary. Physica C, Vol. 460-462, No. 2, September 2007, 1453-1454, ISSN 0921-4534.

Swift, G. (2002). Thermoacoustics: A unifying perspective for some engines and refrigerators, Acoustic Society of America, ISBN 0-7354-0065-2, New York. 
Yang L. W. \& Thummes, G. (2005). High frequency two-stage pulse tube cryocooler with base temperature below 20 K. Cryogenics, Vol. 45, No. 2, February 2005, 155-159, ISSN 0011-2275.

Qiu, L. M.; Sun, D.M.; Yan, W.L.; Chen, P.; Gan, Z.H.; Zhang, X.J.; Chen, G.B. (2005). Investigation on a thermoacoustically driven pulse tube cooler working at $80 \mathrm{~K}$. Cryogenics, Vol. 45, No. 5, May 2005, 380-385, ISSN 0011-2275.

Ruibal, M.; Ferro, G.; Osorio, M. R.; Maza, J.; Veira, J. A.; Vidal, F. (2007). Size effects on the quenching to the normal state of $\mathrm{YBa}_{2} \mathrm{Cu}_{3} \mathrm{O}_{7-\delta}$ thin-film superconductors. Physical Review B, Vol. 75, No. 1, January 2007, 012504(4pp), ISSN 0163-1829.

Viña, J; González, M. T.; Ruibal, M.; Currás, S. R.; Veira, J. A.; Maza, J.; Vidal, F. (2003). Self-heating effects on the transition to a highly dissipative state at high current density in superconducting $\mathrm{YBa}_{2} \mathrm{Cu}_{3} \mathrm{O}_{7-\delta}$ thin films. Physical Review B, Vol. 68, No. 22, December 2003, 224506(10pp), ISSN 0163-1829, and references therein.

Maza, J.; Ferro, G.; Veira J. A.; Vidal, F. (2008). Transition to the normal state induced by high current densities in $\mathrm{YBa}_{2} \mathrm{Cu}_{3} \mathrm{O}_{7-\delta}$ thin films: A thermal runaway account. Physical Review B, Vol. 78, No. 9, September 2008, 094512(9pp), ISSN 0163-1829.

Mosqueira, J.; Cabeza, O.; François, M. X.; Torrón, C.; Vidal, F. (1993). Measurements of pool boiling heat transfer from ceramic $\mathrm{YBa}_{2} \mathrm{Cu}_{3} \mathrm{O}_{7 \text {-delta }}$ superconductors to liquid nitrogen. Superconductor Science and Technology, Vol. 6, No. 8, August 1993, 584-588, ISSN 0953-2048.

Weinstock, H. (Editor). (2000). Applications of Superconductivity (NATO ASI Series E: Applied Sciences - Vol. 365), pp. 247-415, Proceedings of the NATO Advanced Study Institute on Applications of Superconductivity, Loen (Norway), June 1997, Kluwer Academic Publishers, ISBN 0-7923-6113-X, Dordrecht (The Netherlands).

Skocpol, W. J.; Beasley, M. R.; Tinkham, M. (1974). Self-heating hotspots in superconducting thin-film microbridges. Journal of Applied Physics, Vol. 45, No. 9, September 1974, 4054-4066, ISSN 0021-8979.

See e. g., Gurevich, A. VI. \& Mints, R. G. (1987). Self-heating in normal metals and superconductors. Reviews of Modern Physics , Vol. 59, No. 4, December 1987, 941-999, ISSN 0034-6861.

Poulin, G. D.; Lachapelle, J.; Moffat, S. H.; Hegmann, F. A.; Preston, J. S. (1995). Current-voltage characteristics of dc voltage biased high temperature superconducting microbridges. Applied Physics Letters, Vol. 66, No. 19, May 1995, 2576-2578, ISSN 0003-6951. 


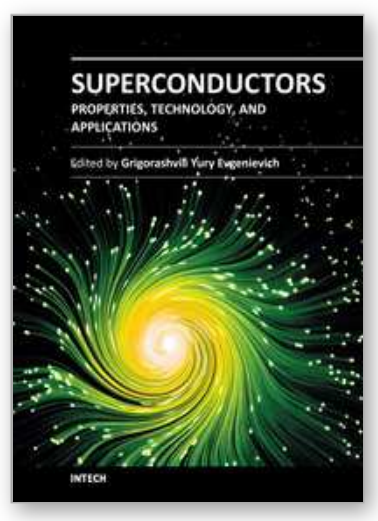

\author{
Superconductors - Properties, Technology, and Applications \\ Edited by Dr. Yury Grigorashvili
}

ISBN 978-953-51-0545-9

Hard cover, 436 pages

Publisher InTech

Published online 20, April, 2012

Published in print edition April, 2012

Book "Superconductors - Properties, Technology, and Applications" gives an overview of major problems encountered in this field of study. Most of the material presented in this book is the result of authors' own research that has been carried out over a long period of time. A number of chapters thoroughly describe the fundamental electrical and structural properties of the superconductors as well as the methods researching those properties. The sourcebook comprehensively covers the advanced techniques and concepts of superconductivity. It's intended for a wide range of readers.

\title{
How to reference
}

In order to correctly reference this scholarly work, feel free to copy and paste the following:

J.A. Veira, M.R. Osorio and F. Vidal (2012). Thermal Behaviour and Refrigeration of High-Temperature Superconducting Fault Current Limiters and Microlimiters, Superconductors - Properties, Technology, and Applications, Dr. Yury Grigorashvili (Ed.), ISBN: 978-953-51-0545-9, InTech, Available from:

http://www.intechopen.com/books/superconductors-properties-technology-and-applications/thermal-behaviourand-refrigeration-of-high-temperature-superconducting-fault-current-limiters-and-m

\section{INTECH}

open science | open minds

\section{InTech Europe}

University Campus STeP Ri

Slavka Krautzeka 83/A

51000 Rijeka, Croatia

Phone: +385 (51) 770447

Fax: +385 (51) 686166

www.intechopen.com

\section{InTech China}

Unit 405, Office Block, Hotel Equatorial Shanghai

No.65, Yan An Road (West), Shanghai, 200040, China

中国上海市延安西路65号上海国际贵都大饭店办公楼405单元

Phone: +86-21-62489820

Fax: +86-21-62489821 
(C) 2012 The Author(s). Licensee IntechOpen. This is an open access article distributed under the terms of the Creative Commons Attribution 3.0 License, which permits unrestricted use, distribution, and reproduction in any medium, provided the original work is properly cited. 